\title{
Fully Discrete Finite Element Approximation for the Stabilized Gauge-Uzawa Method to Solve the Boussinesq Equations
}

\author{
Jae-Hong Pyo \\ Department of Mathematics, Kangwon National University, Chuncheon 200-701, Republic of Korea \\ Correspondence should be addressed to Jae-Hong Pyo; jhpyo@kangwon.ac.kr
}

Received 9 February 2013; Accepted 11 March 2013

Academic Editor: Jinyun Yuan

Copyright (C) 2013 Jae-Hong Pyo. This is an open access article distributed under the Creative Commons Attribution License, which permits unrestricted use, distribution, and reproduction in any medium, provided the original work is properly cited.

\begin{abstract}
The stabilized Gauge-Uzawa method (SGUM), which is a 2nd-order projection type algorithm used to solve Navier-Stokes equations, has been newly constructed in the work of Pyo, 2013. In this paper, we apply the SGUM to the evolution Boussinesq equations, which model the thermal driven motion of incompressible fluids. We prove that SGUM is unconditionally stable, and we perform error estimations on the fully discrete finite element space via variational approach for the velocity, pressure, and temperature, the three physical unknowns. We conclude with numerical tests to check accuracy and physically relevant numerical simulations, the Bénard convection problem and the thermal driven cavity flow.
\end{abstract}

\section{Introduction}

The stabilized Gauge-Uzawa method (SGUM) is a 2nd-order projection type method to solve the evolution Navier-Stokes equations. In this paper, we extend SGUM to the evolution Boussinesq equations: given a bounded polygon $\Omega$ in $\mathbb{R}^{d}$ with $d=2$ or 3 ,

$$
\begin{gathered}
\mathbf{u}_{t}+(\mathbf{u} \cdot \nabla) \mathbf{u}+\nabla p-\mu \Delta \mathbf{u}+\kappa \mu^{2} \mathbf{g} \theta=\mathbf{f}, \quad \text { in } \Omega, \\
\nabla \cdot \mathbf{u}=0, \quad \text { in } \Omega, \\
\theta_{t}+\mathbf{u} \cdot \nabla \theta-\lambda \mu \Delta \theta=b, \quad \text { in } \Omega,
\end{gathered}
$$

with initial conditions $\mathbf{u}(\mathbf{x}, 0)=\mathbf{u}^{0}, \theta(\mathbf{x}, 0)=\theta^{0}$ in $\Omega$, vanishing Dirichlet boundary conditions $\mathbf{u}=0, \theta=0$ on $\partial \Omega$, and pressure mean-value $\int_{\Omega} p=0$. The forcing functions $\mathbf{f}$ and $b$ are given, and $\mathbf{g}$ is the vector of gravitational acceleration. The nondimensional numbers $\mu=\operatorname{Re}^{-1}$ and $\lambda=\operatorname{Pr}^{-1}$ are reciprocal of the Reynolds and Prandtl numbers, respectively, whereas $\kappa$ is the Grashof number. The Boussinesq system (1) describes fluid motion due to density differences which are in turn induced by temperature gradients: hot and thus less dense fluid tends to rise against gravity and cooler fluid falls in its place. The simplest governing equations are thus the Navier-Stokes equations for motion of an incompressible fluid, with forcing $\kappa \mu^{2} \mathbf{g} \theta$ due to buoyancy and the heat equation for diffusion and transport of heat. Density differences are thus ignored altogether except for buoyancy.

The projection type methods are representative solvers for the incompressible flows, and the Gauge-Uzawa method is a typical projection method. The Gauge-Uzawa method was constructed in [1] to solve Navier-Stokes equations and extended to more complicated problems, the Boussinesq equations in [2] and the nonconstant density Navier-Stokes equations in [3]. However, most of studies for the GaugeUzawa method have been limited only for the first-order accuracy backward Euler time marching algorithm. The second-order Gauge-Uzawa method using BDF2 scheme was introduced in [4] and proved superiority for accuracy on the normal mode space, but we could not get any theoretical proof via energy estimate even stability and we suffer from weak stability performance on the numerical test. Recently, we construct SGUM in [5] which is unconditionally stable for semidiscrete level to solve the Navier-Stokes equations. The goal of this paper is to extend SGUM to the Boussinesq equations (1), which model the motion of an incompressible viscous fluid due to thermal effects [6,7]. We will estimate errors and stability on the fully discrete finite element space. The main difficulties in the fully discrete estimation arise from losing the cancellation law due to the failing of 
the divergence free condition of the discrete velocity function. The strategy of projection type methods computes first an artificial velocity and then decomposes it to divergence free velocity and curl free functions. However, the divergence free condition cannot be preserved in discrete finite element space, and so the cancellation law (12) can not be satisfied any more. In order to solve this difficulty, we impose the discontinuous velocity on across interelement boundaries to make fulfill discrete divergence free velocity (12) automatically. We will discuss this issue at Remark 2 below. This discontinuity makes it difficult to treat nonlinear term and to apply the integration by parts, because the discontinuous solution is not included in $H^{1}(\Omega)$. So we need to hire technical skills in proof of this paper.

One more remarkable discovery is in the second numerical test at the last section which is the Bénard convection problem with the same setting in [2]. In this performance, we newly find out that the number of circulations depends on the time step size $\tau$. We obtain similar simulation within [2] for a relatively larger $\tau$, but the behavior is changed for the small $\tau$. So we conclude that the numerical result of the Bénard convection problem in [2] is not an eventual solution and a more smaller time marching step is required to get the desired simulation.

We will denote $\tau$ as the time marching size. Also we will use $\delta$ as the difference of 2 consecutive functions for example, for any sequence function $z^{n+1}$,

$$
\begin{gathered}
\delta z^{n+1}=z^{n+1}-z^{n} \\
\delta \delta z^{n+1}=\delta\left(\delta z^{n+1}\right)=z^{n+1}-2 z^{n}+z^{n-1}, \ldots
\end{gathered}
$$

In order to introduce the finite element discretization, we need further notations. Let $H^{s}(\Omega)$ be the Sobolev space with $s$ derivatives in $L^{2}(\Omega)$, set $\mathbf{L}^{2}(\Omega)=\left(L^{2}(\Omega)\right)^{d}$ and $\mathbf{H}^{s}(\Omega)=$ $\left(H^{s}(\Omega)\right)^{d}$, where $d=2$ or 3 , and denote by $L_{0}^{2}(\Omega)$ the subspace of $L^{2}(\Omega)$ of functions with vanishing mean value. We indicate with $\|\cdot\|_{s}$ the norm in $H^{s}(\Omega)$ and with $\langle\cdot, \cdot\rangle$ the inner product in $L^{2}(\Omega)$. Let $\mathfrak{I}=\{K\}$ be a shaperegular quasiuniform partition of $\Omega$ of mesh size $h$ into closed elements $K[8-10]$. The vector and scalar finite element spaces are

$$
\begin{gathered}
\mathbb{W}_{h}:=\left\{\mathbf{w}_{h} \in \mathbf{L}^{2}(\Omega):\left.\mathbf{w}_{h}\right|_{K} \in \mathscr{P}(K) \forall K \in \mathfrak{T}\right\}, \\
\mathbb{V}_{h}:=\mathbb{W}_{h} \cap \mathbf{H}_{0}^{1}(\Omega), \\
\mathbb{T}_{h}:=\left\{\theta_{h} \in H_{0}^{1}(\Omega) \cap C^{0}(\Omega):\left.\theta_{h}\right|_{K} \in \mathscr{Q}(K) \forall K \in \mathfrak{I}\right\}, \\
\mathbb{P}_{h}:=\left\{q_{h} \in L_{0}^{2}(\Omega) \cap C^{0}(\Omega):\left.q_{h}\right|_{K} \in \mathscr{R}(K) \forall K \in \mathfrak{T}\right\},
\end{gathered}
$$

where $\mathscr{P}(K), \mathcal{Q}(K)$, and $\mathscr{R}(K)$ are spaces of polynomials with degree bounded uniformly with respect to $K \in \mathfrak{I}[9,10]$. We stress that the space $\mathbb{P}_{h}$ is composed of continuous functions to ensure the crucial equality

$$
\left\langle\nabla \cdot \mathbf{w}_{h}, \phi_{h}\right\rangle=-\left\langle\mathbf{w}_{h}, \nabla \phi_{h}\right\rangle, \quad \forall \mathbf{w}_{h} \in \mathbb{V}_{h}, \forall \phi_{h} \in \mathbb{P}_{h}
$$

Using the following discrete counterpart of the form $\mathcal{N}(\mathbf{u}, \mathbf{v}, \mathbf{w}):=\langle(\mathbf{u} \cdot \nabla) \mathbf{v}, \mathbf{w}\rangle$

$$
\begin{aligned}
\mathcal{N}\left(\mathbf{u}_{h}, \mathbf{v}_{h}, \mathbf{w}_{h}\right) & :=\frac{1}{2}\left\langle\left(\mathbf{u}_{h} \cdot \nabla\right) \mathbf{v}_{h}, \mathbf{w}_{h}\right\rangle \\
& -\frac{1}{2}\left\langle\left(\mathbf{u}_{h} \cdot \nabla\right) \mathbf{w}_{h}, \mathbf{v}_{h}\right\rangle,
\end{aligned}
$$

we now introduce the fully discrete SGUM to solve the evolution Boussinesq equations (1).

Algorithm 1 (the fully discrete stabilized Gauge-Uzawa method). Compute $\theta_{h}^{1}, \mathbf{u}_{h}^{1}$, and $p_{h}^{1}$ via any method satisfying Assumption 4 below, and set $\psi_{h}^{1}=(-2 \tau / 3) p_{h}^{1}$ and $q_{h}^{1}=0$. Repeat for $1 \leq n \leq N=[T / \tau-1]$.

Step 1. Set $\mathbf{u}_{h}^{*}=2 \mathbf{u}_{h}^{n}-\mathbf{u}_{h}^{n-1}$ and $\theta_{h}^{*}=2 \theta_{h}^{n}-\theta_{h}^{n-1}$, and then find $\widehat{\mathbf{u}}_{h}^{n+1} \in \mathbb{V}_{h}$ as the solution of

$$
\begin{aligned}
\frac{1}{2 \tau}\langle & \left.3 \widehat{\mathbf{u}}_{h}^{n+1}-4 \mathbf{u}_{h}^{n}+\mathbf{u}_{h}^{n-1}, \mathbf{w}_{h}\right\rangle \\
& +\left\langle\nabla p_{h}^{n}, \mathbf{w}_{h}\right\rangle+\mathcal{N}\left(\mathbf{u}_{h}^{*}, \widehat{\mathbf{u}}_{h}^{n+1}, \mathbf{w}_{h}\right) \\
& +\mu\left\langle\nabla \widehat{\mathbf{u}}_{h}^{n+1}, \nabla \mathbf{w}_{h}\right\rangle+\kappa \mu^{2}\left\langle\mathbf{g} \theta_{h}^{*}, \mathbf{w}_{h}\right\rangle \\
= & \left\langle\mathbf{f}\left(t^{n+1}\right), \mathbf{w}_{h}\right\rangle, \quad \forall \mathbf{w}_{h} \in \mathbb{V}_{h} .
\end{aligned}
$$

Step 2. Find $\psi_{h}^{n+1} \in \mathbb{P}_{h}$ as the solution of

$$
\begin{array}{r}
\left\langle\nabla \psi_{h}^{n+1}, \nabla \phi_{h}\right\rangle=\left\langle\nabla \psi_{h}^{n}, \nabla \phi_{h}\right\rangle+\left\langle\nabla \cdot \widehat{\mathbf{u}}_{h}^{n+1}, \phi_{h}\right\rangle, \\
\forall \phi_{h} \in \mathbb{P}_{h} .
\end{array}
$$

Step 3. Update $\mathbf{u}_{h}^{n+1}$ and $q_{h}^{n+1} \in \mathbb{P}_{h}$ according to

$$
\begin{gathered}
\mathbf{u}_{h}^{n+1}=\widehat{\mathbf{u}}_{h}^{n+1}+\nabla\left(\psi_{h}^{n+1}-\psi_{h}^{n}\right), \\
\left\langle q_{h}^{n+1}, \phi_{h}\right\rangle=\left\langle q_{h}^{n}, \phi_{h}\right\rangle-\left\langle\nabla \cdot \widehat{\mathbf{u}}_{h}^{n+1}, \phi_{h}\right\rangle, \quad \forall \phi_{h} \in \mathbb{P}_{h} .
\end{gathered}
$$

Step 4. Update pressure $p_{h}^{n+1}$ by

$$
p_{h}^{n+1}=-\frac{3 \psi_{h}^{n+1}}{2 \tau}+\mu q_{h}^{n+1} .
$$

Step 5. Find $\theta_{h}^{n+1} \in \mathbb{T}_{h}$ as the solution of

$$
\begin{aligned}
\frac{1}{2 \tau}\langle & \left.3 \theta_{h}^{n+1}-4 \theta_{h}^{n}+\theta_{h}^{n-1}, \phi_{h}\right\rangle \\
& +\mathcal{N}\left(\mathbf{u}_{h}^{n+1}, \theta_{h}^{n+1}, \phi_{h}\right) \\
& +\lambda \mu\left\langle\nabla \theta_{h}^{n+1}, \nabla \phi_{h}\right\rangle \\
= & \left\langle b\left(t^{n+1}\right), \phi_{h}\right\rangle, \quad \forall \phi_{h} \in \mathbb{T}_{h} .
\end{aligned}
$$

Remark 2 (discontinuity of $\mathbf{u}_{h}^{n+1}$ ). We note that $\mathbf{u}_{h}^{n+1}$ is a discontinuous function across inter-element boundaries and 
that, in light of (7) and (8), $\mathbf{u}_{h}^{n+1}$ is discrete divergence free in the sense that

$$
\left\langle\mathbf{u}_{h}^{n+1}, \nabla \phi_{h}\right\rangle=\left\langle\widehat{\mathbf{u}}_{h}^{n+1}+\delta \nabla \psi_{h}^{n+1}, \nabla \phi_{h}\right\rangle=0, \quad \forall \phi_{h} \in \mathbb{P}_{h} .
$$

We now summarize the results of this paper along with organization. We introduce appropriate Assumptions 1-5 in Section 2 and introduce well-known lemmas. In Section 3, we prove the stability result.

Theorem 3 (stability). The SGUM is unconditionally stable in the sense that, for all $\tau>0$, the following a priori bound holds:

$$
\begin{aligned}
& \left\|\mathbf{u}_{h}^{N+1}\right\|_{0}^{2}+\left\|\widehat{\mathbf{u}}_{h}^{N+1}\right\|_{0}^{2}+\left\|2 \mathbf{u}_{h}^{N+1}-\mathbf{u}_{h}^{N}\right\|_{0}^{2} \\
& +\left\|2 \theta_{h}^{N+1}-\theta_{h}^{N}\right\|_{0}^{2}+\left\|\theta_{h}^{N+1}\right\|_{0}^{2} \\
& \quad+\sum_{n=1}^{N}\left(\left\|\delta \delta \mathbf{u}_{h}^{n+1}\right\|_{0}^{2}+\left\|\delta \delta \theta_{h}^{n+1}\right\|_{0}^{2}+\left\|\nabla \delta \psi_{h}^{n+1}\right\|_{0}^{2}\right) \\
& +3\left\|\nabla \psi_{h}^{N+1}\right\|_{0}^{2}+2 \mu \tau\left\|q_{h}^{N+1}\right\|_{0}^{2} \\
& +\mu \tau \sum_{n=1}^{N}\left(\left\|\nabla \widehat{\mathbf{u}}_{h}^{n+1}\right\|_{0}^{2}+\lambda\left\|\nabla \theta_{h}^{n+1}\right\|\right) \\
& \leq\left\|\mathbf{u}_{h}^{1}\right\|_{0}^{2}+\left\|2 \mathbf{u}_{h}^{1}-\mathbf{u}_{h}^{0}\right\|_{0}^{2}+\left\|2 \theta_{h}^{1}-\theta_{h}^{0}\right\|_{0}^{2} \\
& +\left\|\theta_{h}^{1}\right\|_{0}^{2}+3\left\|\nabla \psi_{h}^{1}\right\|_{0}^{2}+2 \mu \tau\left\|q_{h}^{1}\right\|_{0}^{2} \\
& +C \tau \sum_{n=1}^{N}\left(\left\|\mathbf{f}\left(t^{n+1}\right)\right\|_{-1}^{2}+\left\|b\left(t^{n+1}\right)\right\|_{-1}^{2}\right) .
\end{aligned}
$$

We then will carry out the following optimal error estimates through several lemmas in Section 4.

Theorem 4 (error estimates). Suppose the exact solution of (1) is smooth enough and $\tau \leq$ Ch. If Assumptions 1, 3, 4, and 5 mentioned later hold, then the errors of Algorithm 1 will be bound by

$$
\begin{gathered}
\tau \sum_{n=1}^{N}\left(\left\|\mathbf{u}\left(t^{n+1}\right)-\mathbf{u}_{h}^{n+1}\right\|_{0}^{2}+\left\|\mathbf{u}\left(t^{n+1}\right)-\widehat{\mathbf{u}}_{h}^{n+1}\right\|_{0}^{2}\right) \\
\leq C\left(\tau^{4}+h^{4}\right), \\
\tau \sum_{n=1}^{N}\left(\left\|\mathbf{u}\left(t^{n+1}\right)-\widehat{\mathbf{u}}_{h}^{n+1}\right\|_{1}^{2}+h^{2}\left\|\theta\left(t^{n+1}\right)-\theta_{h}^{n+1}\right\|_{1}^{2}\right) \\
\leq C\left(\tau^{2}+h^{2}\right), \\
\tau \sum_{n=1}^{N}\left\|\theta\left(t^{n+1}\right)-\theta_{h}^{n+1}\right\|_{0}^{2} \leq C\left(\tau^{4}+h^{4}\right) .
\end{gathered}
$$

Moreover, if Assumption 2 also hold, then one has

$$
\tau \sum_{n=1}^{N}\left\|p\left(t^{n+1}\right)-p_{h}^{n+1}\right\|_{0}^{2} \leq C\left(\tau^{2}+h^{2}\right) .
$$

We note that the condition $\tau \leq C h$ in Theorem 4 can be omitted for the linearized Boussinesq equations (see Remark 16). Finally, we perform numerical tests in Section 5 to check accuracy and physically relevant numerical simulations, the Bénard convection problem and the thermal driven cavity flow.

\section{Preliminaries}

In this section, we introduce 5 assumptions and well known lemmas to use in proof of main theorems. We resort to a duality argument for

$$
-\Delta \mathbf{v}+\nabla r=\mathbf{z}, \quad \nabla \cdot \mathbf{v}=0, \quad \text { in } \Omega,
$$

which is the stationary Stokes system with vanishing boundary condition $\mathbf{v}=\mathbf{0}$, as well as Poisson's equation

$$
-\Delta \omega=\xi, \quad \text { in } \Omega,
$$

with boundary condition $\omega=0$.

We now state a basic assumption about $\Omega$.

Assumption 1 (regularity of $(\mathbf{v}, r)$ and $\omega$ ). The unique solutions $\{\mathbf{v}, r\}$ of (16) and $\omega$ of (17) satisfy

$$
\|\mathbf{v}\|_{2}+\|r\|_{1} \leq C\|\mathbf{z}\|_{0}, \quad\|\omega\|_{2} \leq C\|\xi\|_{0} .
$$

We remark that the validity of Assumption 1 is known if $\partial \Omega$ is of class $\mathbf{C}^{2}[11,12]$, or if $\partial \Omega$ is a two-dimensional convex polygon [13] and is generally believed for convex polyhedral [12].

We impose the following properties for relations between the spaces $\mathbb{V}_{h}$ and $\mathbb{P}_{h}$.

Assumption 2 (discrete inf-sup). There exists a constant $\beta>0$ such that

$$
\inf _{s_{h} \in \mathbb{P}_{h}} \sup _{\mathbf{w}_{h} \in \mathbb{V}_{h}} \frac{\left\langle\nabla \cdot \mathbf{w}_{h}, s_{h}\right\rangle}{\left\|\mathbf{w}_{h}\right\|_{1}\left\|s_{h}\right\|_{0}} \geq \beta .
$$

Assumption 3 (shape regularity and quasiuniformity [8-10]). There exists a constant $C>0$ such that the ratio between the diameter $h_{K}$ of an element $K \in \mathfrak{I}$ and the diameter of the largest ball contained in $K$ is bounded uniformly by $C$, and $h_{K}$ is comparable with the meshsize $h$ for all $K \in \mathfrak{T}$.

In order to launch Algorithm 1, we need to set $\left(\mathbf{u}_{h}^{1}, p_{h}^{1}, \theta_{h}^{1}\right)$ via any first-order methods which hold the following conditions.

Assumption 4 (the setting of the first step values). Let $\left(\mathbf{u}\left(t^{1}\right), p\left(t^{1}\right), \theta\left(t^{1}\right)\right)$ be the exact solution of (1) at $t=t^{1}$. The first step value $\left(\mathbf{u}_{h}^{1}, p_{h}^{1}, \theta_{h}^{1}\right)$ satisfies

$$
\begin{gathered}
\left\|\mathbf{u}\left(t^{1}\right)-\mathbf{u}_{h}^{1}\right\|_{0}+\left\|\theta\left(t^{1}\right)-\theta_{h}^{1}\right\|_{0} \leq C\left(\tau^{2}+h^{2}\right), \\
\left\|\mathbf{u}\left(t^{1}\right)-\mathbf{u}_{h}^{1}\right\|_{1}+\left\|p\left(t^{1}\right)-p_{h}^{1}\right\|_{1}+\left\|\theta\left(t^{1}\right)-\theta_{h}^{1}\right\|_{1} \leq C(\tau+h) .
\end{gathered}
$$


Assumption 5 (approximability [8-10]). For each $(\mathbf{w}, \eta, s) \in$ $\mathbf{H}^{2}(\Omega) \times H^{2}(\Omega) \times H^{1}(\Omega)$, there exist approximations $\left(\mathbf{w}_{h}, \eta_{h}, s_{h}\right) \in \mathbb{V}_{h} \times \mathbb{T}_{h} \times \mathbb{P}_{h}$ such that

$$
\begin{gathered}
\left\|\mathbf{w}-\mathbf{w}_{h}\right\|_{0}+h\left\|\mathbf{w}-\mathbf{w}_{h}\right\|_{1} \leq C h^{2}\|\mathbf{w}\|_{2}, \\
\left\|\eta-\eta_{h}\right\|_{0}+h\left\|\eta-\eta_{h}\right\|_{1} \leq C h^{2}\|\eta\|_{2}, \\
\left\|s-s_{h}\right\|_{0} \leq C h\|s\|_{1} .
\end{gathered}
$$
in [14].

The following elementary but crucial relations are derived

Lemma 5 (inverse inequality). If $I_{h}$ denotes the Clement interpolant, then

$$
\begin{gathered}
\left\|I_{h} \mathbf{w}\right\|_{\mathbf{L}^{3}(\Omega)} \leq C h^{-d / 6}\left\|I_{h} \mathbf{w}\right\|_{0}, \\
\left\|\mathbf{w}-I_{h} \mathbf{w}\right\|_{\mathbf{L}^{3}(\Omega)} \leq C h^{2-d / 6}\|\mathbf{w}\|_{2} .
\end{gathered}
$$

Lemma $\mathbf{6}$ (div-grad relation). If $\mathbf{w} \in \mathbf{H}_{0}^{1}(\Omega)$, then

$$
\|\nabla \cdot \mathbf{w}\|_{0} \leq\|\nabla \mathbf{w}\|_{0} .
$$

Let now $\left(\mathbf{v}_{h}, r_{h}\right) \in \mathbb{V}_{h} \times \mathbb{P}_{h}$ indicate the finite element solution of (16); namely,

$$
\begin{gathered}
\left\langle\nabla \mathbf{v}_{h}, \nabla \mathbf{w}_{h}\right\rangle+\left\langle\nabla r_{h}, \mathbf{w}_{h}\right\rangle=\left\langle\mathbf{z}, \mathbf{w}_{h}\right\rangle, \quad \forall \mathbf{w}_{h} \in \mathbb{V}_{h}, \\
\left\langle\nabla \cdot \mathbf{v}_{h}, s_{h}\right\rangle=0, \quad \forall s_{h} \in \mathbb{P}_{h} .
\end{gathered}
$$

Then we can find the well-known lemmas in [8-10].

Lemma 7 (error estimates for mixed FEM). Let $(\mathbf{v}, r) \in$ $\mathbf{H}_{0}^{1}(\Omega) \times L_{0}^{2}(\Omega)$ be the solutions of $(16)$, and, $\left(\mathbf{v}_{h}, r_{h}\right)=$ $\mathbb{S}_{h}(\mathbf{v}, r) \in \mathbb{V}_{h} \times \mathbb{P}_{h}$ be the Stokes projection defined by (24), respectively. If Assumptions 1, 2, 3, and 5 hold, then

$$
\begin{gathered}
\left\|\mathbf{v}-\mathbf{v}_{h}\right\|_{0}+h\left\|\mathbf{v}-\mathbf{v}_{h}\right\|_{1}+h\left\|r-r_{h}\right\|_{0} \leq C h^{2}\left(\|\mathbf{v}\|_{2}+\|r\|_{1}\right), \\
\left\|\left|\mathbf{v}-\mathbf{v}_{h}\right|\right\|:=\left\|\mathbf{v}-\mathbf{v}_{h}\right\|_{L^{\infty}(\Omega)}+\left\|\nabla\left(\mathbf{v}-\mathbf{v}_{h}\right)\right\|_{\mathbf{L}^{3}(\Omega)} \leq C\|\mathbf{z}\|_{0} .
\end{gathered}
$$

Proof. Inequality (25) is standard [8-10]. To establish (26), we just deal with the $\mathbf{L}^{\infty}$-norm, since the other can be treated similarly. If $I_{h}$ denotes the Clément interpolant, then $\left\|\mathbf{v}-I_{h} \mathbf{v}\right\|_{\mathbf{L}^{\infty}(\Omega)} \leq C\|\mathbf{v}\|_{2}$ and

$$
\left\|I_{h} \mathbf{v}-\mathbf{v}_{h}\right\|_{\mathbf{L}^{\infty}(\Omega)} \leq C h^{-d / 2}\left\|I_{h} \mathbf{v}-\mathbf{v}_{h}\right\|_{\mathbf{L}^{2}(\Omega)} \leq C\|\mathbf{v}\|_{2}
$$

as a consequence of an inverse estimate. This completes the proof.

Remark $8\left(H^{1}\right.$ stability of $\left.r_{h}\right)$. The bound $\left\|\nabla r_{h}\right\|_{0} \leq C\left(\|\mathbf{v}\|_{2}+\right.$ $\left.\|r\|_{1}\right)$ is a simple by-product of (16). To see this, we add and subtract $I_{h} q$, use the stability of $I_{h}$ in $H^{1}$, and observe that (25) implies $\left\|\nabla\left(r_{h}-I_{h} r\right)\right\|_{0} \leq C h^{-1}\left\|r_{h}-I_{h} r\right\|_{0} \leq C$.
Lemma 9 (error estimates for Poisson's equation $[8,10])$. Let $\omega \in H_{0}^{1}(\Omega)$ be the solution of Poisson's equation (17), and, $\omega_{h} \in$ $\mathbb{T}_{h}$ be its finite element approximation

$$
\left\langle\nabla \omega_{h}, \nabla \phi_{h}\right\rangle=\left\langle\xi, \phi_{h}\right\rangle, \quad \forall \phi_{h} \in \mathbb{T}_{h} .
$$

If Assumptions 1, 3, and 5 hold, then there exists a positive constant $C$ satisfying

$$
\begin{aligned}
&\left\|\omega-\omega_{h}\right\|_{0}+h\left\|\omega-\omega_{h}\right\|_{1} \leq C h^{2}\|\omega\|_{2} \\
& \leq C h^{2}\|\xi\|_{0}, \\
&\left\|\left|\omega-\omega_{h}\right|\right\| \leq C\|\xi\|_{0} .
\end{aligned}
$$

We finally state without proof several properties of the nonlinear form $\mathcal{N}$. In view of (5), we have the following properties of $\mathcal{N}$ for all $\mathbf{u}_{h}, \mathbf{v}_{h}, \mathbf{w}_{h} \in \mathbb{V}_{h}$ :

$$
\begin{gathered}
\mathcal{N}\left(\mathbf{u}_{h}, \mathbf{v}_{h}, \mathbf{w}_{h}\right)=-\mathcal{N}\left(\mathbf{u}_{h}, \mathbf{w}_{h}, \mathbf{v}_{h}\right), \\
\mathcal{N}\left(\mathbf{u}_{h}, \mathbf{v}_{h}, \mathbf{v}_{h}\right)=0, \\
\nabla \cdot \mathbf{u}=0 \Longrightarrow \mathcal{N}\left(\mathbf{u}, \mathbf{v}_{h}, \mathbf{w}_{h}\right) \\
=\left\langle(\mathbf{u} \cdot \nabla) \mathbf{v}_{h}, \mathbf{w}_{h}\right\rangle \\
=-\left\langle(\mathbf{u} \cdot \nabla) \mathbf{w}_{h}, \mathbf{v}_{h}\right\rangle .
\end{gathered}
$$

Applying Sobolev imbedding lemma yields the following useful results.

Lemma 10 (bounds on nonlinear convection $[1,12])$. Let $\mathbf{u}, \mathbf{v} \in \mathbf{H}^{2}(\Omega)$ with $\nabla \cdot \mathbf{u}=0$, and let $\mathbf{u}_{h}, \mathbf{v}_{h}, \mathbf{w}_{h} \in \mathbb{V}_{h}$. Then

$$
\begin{gathered}
\mathcal{N}\left(\mathbf{u}, \mathbf{v}_{h}, \mathbf{w}_{h}\right) \leq C\left\{\begin{array}{l}
\|\mathbf{u}\|_{1}\left\|\mathbf{v}_{h}\right\|_{1}\left\|\mathbf{w}_{h}\right\|_{1} \\
\|\mathbf{u}\|_{2}\left\|\mathbf{v}_{h}\right\|_{1}\left\|\mathbf{w}_{h}\right\|_{0} \\
\|\mathbf{u}\|_{2}\left\|\mathbf{v}_{h}\right\|_{0}\left\|\mathbf{w}_{h}\right\|_{1},
\end{array}\right. \\
\mathcal{N}\left(\mathbf{u}_{h}, \mathbf{v}, \mathbf{w}_{h}\right) \leq C\left\|\mathbf{u}_{h}\right\|_{0}\|\mathbf{v}\|_{2}\left\|\mathbf{w}_{h}\right\|_{1} .
\end{gathered}
$$

In addition,

$$
\mathcal{N}\left(\mathbf{u}_{h}, \mathbf{v}_{h}, \mathbf{w}_{h}\right) \leq C\left\{\begin{array}{c}
\left\|\mathbf{u}_{h}\right\|_{0}\left\|\left|\mathbf{v}_{h}\right|\right\|\left\|\mathbf{w}_{h}\right\|_{1} \\
\left\|\mathbf{u}_{h}\right\|_{\mathbf{L}^{3}(\Omega)}\left\|\mathbf{v}_{h}\right\|\left\|_{1}\right\| \mathbf{w}_{h} \|_{1} .
\end{array}\right.
$$

Remark 11 (Treatment of convection term). As we mention in Remark $2, \mathbf{u}_{h}^{n+1}$ is discontinuous across inter-element boundaries and so $\mathbf{u}_{h}^{n+1} \notin \mathbf{H}^{1}(\Omega)$. Thus, we can't directly apply (32) anymore to treat the convection term. To solve this difficulty, we apply (34) together with (26) and inverse inequality Lemma 5.

We will use the following algebraic identities frequently to treat time derivative terms. 
Lemma 12 (inner product of time derivative terms). For any sequence $\left\{z^{n}\right\}_{n=0}^{N}$, one has

$$
\begin{aligned}
& 2\left\langle 3 z^{n+1}-4 z^{n}+z^{n-1}, z^{n+1}\right\rangle \\
& =\delta\left\|z^{n+1}\right\|_{0}^{2}+\delta\left\|2 z^{n+1}-z^{n}\right\|_{0}^{2}+\left\|\delta \delta z^{n+1}\right\|_{0}^{2}, \\
& 2\left\langle z^{n+1}-z^{n}, z^{n+1}\right\rangle \\
& =\left\|z^{n+1}\right\|_{0}^{2}-\left\|z^{n}\right\|_{0}^{2}+\left\|z^{n+1}-z^{n}\right\|_{0}^{2}, \\
& 2\left\langle z^{n+1}-z^{n}, z^{n}\right\rangle \\
& =\left\|z^{n+1}\right\|_{0}^{2}-\left\|z^{n}\right\|_{0}^{2}-\left\|z^{n+1}-z^{n}\right\|_{0}^{2} .
\end{aligned}
$$

\section{Proof of Stability}

We show that the SGUM is unconditionally stable via a standard energy method in this section. We start to prove stability with rewriting the momentum equation (6) by using (8) and (10) as follows:

$$
\begin{aligned}
\frac{1}{2 \tau}\left\langle 3 \mathbf{u}_{h}^{n+1}-4 \mathbf{u}_{h}^{n}+\mathbf{u}_{h}^{n-1}, \mathbf{w}_{h}\right\rangle \\
+\mathcal{N}\left(\mathbf{u}_{h}^{*}, \widehat{\mathbf{u}}_{h}^{n+1}, \mathbf{w}_{h}\right)+\kappa \mu^{2}\left\langle\mathbf{g} \theta_{h}^{*}, \mathbf{w}_{h}\right\rangle \\
\quad-\left\langle\nabla\left(\frac{3}{2 \tau} \psi_{h}^{n+1}-\mu q_{h}^{n}\right), \mathbf{w}_{h}\right\rangle \\
+\mu\left\langle\nabla \widehat{\mathbf{u}}_{h}^{n+1}, \nabla \mathbf{w}_{h}\right\rangle=\left\langle\mathbf{f}\left(t^{n+1}\right), \mathbf{w}_{h}\right\rangle .
\end{aligned}
$$

We now choose $\mathbf{w}_{h}=4 \tau \widehat{\mathbf{u}}_{h}^{n+1}$ and apply (35) to obtain

$$
\begin{aligned}
\delta\left\|\mathbf{u}_{h}^{n+1}\right\|_{0}^{2}+\delta\left\|2 \mathbf{u}_{h}^{n+1}-\mathbf{u}_{h}^{n}\right\|_{0}^{2} \\
\quad+\left\|\delta \delta \mathbf{u}_{h}^{n+1}\right\|_{0}^{2}+4 \tau \mu\left\|\nabla \widehat{\mathbf{u}}_{h}^{n+1}\right\|_{0}^{2} \\
=\sum_{i=1}^{4} A_{i},
\end{aligned}
$$

where

$$
\begin{gathered}
A_{1}:=6\left\langle\nabla \psi_{h}^{n+1}, \widehat{\mathbf{u}}_{h}^{n+1}\right\rangle, \\
A_{2}:=4 \tau \mu\left\langle q_{h}^{n}, \nabla \cdot \widehat{\mathbf{u}}_{h}^{n+1}\right\rangle, \\
A_{3}:=4 \tau\left\langle\mathbf{f}\left(t^{n+1}\right), \widehat{\mathbf{u}}_{h}^{n+1}\right\rangle, \\
A_{4}:=-4 \tau \kappa \mu^{2}\left\langle\mathbf{g}\left(2 \theta_{h}^{n}-\theta_{h}^{n-1}\right), \widehat{\mathbf{u}}_{h}^{n+1}\right\rangle .
\end{gathered}
$$

We give thanks to (30) for eliminating the convection term. In light of $\widehat{\mathbf{u}}_{h}^{n+1}=\mathbf{u}_{h}^{n+1}-\nabla \delta \psi_{h}^{n+1}$, (12) and (36) yield

$$
\begin{aligned}
A_{1} & =-6\left\langle\nabla \psi_{h}^{n+1}, \nabla \delta \psi_{h}^{n+1}\right\rangle \\
& =-3\left(\left\|\nabla \psi_{h}^{n+1}\right\|_{0}^{2}-\left\|\nabla \psi_{h}^{n}\right\|_{0}^{2}+\left\|\nabla \delta \psi_{h}^{n+1}\right\|_{0}^{2}\right) .
\end{aligned}
$$

Before we estimate $A_{2}$, we evaluate an inequality via choosing $\phi_{h}=\delta q_{h}^{n+1}$ in (9) to get

$$
\begin{aligned}
\left\|\delta q_{h}^{n+1}\right\|_{0}^{2} & =-\left\langle\nabla \cdot \widehat{\mathbf{u}}_{h}^{n+1}, \delta q_{h}^{n+1}\right\rangle \\
& \leq\left\|\nabla \cdot \widehat{\mathbf{u}}_{h}^{n+1}\right\|_{0}\left\|\delta q_{h}^{n+1}\right\|_{0} .
\end{aligned}
$$

Lemma 6 derives $\left\|\delta q_{h}^{n+1}\right\|_{0}^{2} \leq\left\|\nabla \cdot \widehat{\mathbf{u}}_{h}^{n+1}\right\|_{0}^{2} \leq\left\|\nabla \widehat{\mathbf{u}}_{h}^{n+1}\right\|_{0}^{2}$, and so (9) and (37) lead us to

$$
\begin{aligned}
A_{2} & =-4 \mu \tau\left\langle q_{h}^{n}, \delta q_{h}^{n+1}\right\rangle \\
& =-2 \mu \tau\left(\left\|q_{h}^{n+1}\right\|_{0}^{2}-\left\|q_{h}^{n}\right\|_{0}^{2}-\left\|\delta q_{h}^{n+1}\right\|_{0}^{2}\right) \\
& \leq-2 \mu \tau\left(\left\|q_{h}^{n+1}\right\|_{0}^{2}-\left\|q_{h}^{n}\right\|_{0}^{2}\right)+2 \mu \tau\left\|\nabla \widehat{\mathbf{u}}_{h}^{n+1}\right\|_{0}^{2} .
\end{aligned}
$$

Clearly, we have

$$
A_{3} \leq C \frac{\tau}{\mu}\left\|\mathbf{f}\left(t^{n+1}\right)\right\|_{-1}^{2}+\tau \mu\left\|\nabla \widehat{\mathbf{u}}_{h}^{n+1}\right\|_{0}^{2} .
$$

We now attack $A_{4}$ with $\left\|\widehat{\mathbf{u}}_{h}^{n+1}\right\|_{0}^{2}=\left\|\mathbf{u}_{h}^{n+1}\right\|_{0}^{2}+\left\|\nabla \delta \psi_{h}^{n+1}\right\|_{0}^{2}$ which comes from (8) and (12). Then we arrive at

$$
\begin{aligned}
A_{4} \leq & C \kappa^{2} \mu^{4} \tau\left\|2 \theta_{h}^{n}-\theta_{h}^{n-1}\right\|_{0}^{2} \\
& +C \tau\left\|\mathbf{u}_{h}^{n+1}\right\|_{0}^{2}+\left\|\nabla \delta \psi_{h}^{n+1}\right\|_{0}^{2} .
\end{aligned}
$$

In conjunction with $\mathcal{N}\left(\mathbf{u}_{h}^{*}, \theta_{h}^{n+1}, \theta_{h}^{n+1}\right)=0$ which comes from (30), if we choose $\phi_{h}=4 \tau \theta_{h}^{n+1}$ in (11) and use (35), then we obtain

$$
\begin{aligned}
\delta\left\|\theta_{h}^{n+1}\right\|_{0}^{2} & +\delta\left\|2 \theta_{h}^{n+1}-\theta_{h}^{n}\right\|_{0}^{2} \\
& +\left\|\delta \delta \theta_{h}^{n+1}\right\|_{0}^{2}+4 \tau \lambda \mu\left\|\nabla \theta_{h}^{n+1}\right\| \\
= & 4 \tau\left\langle b\left(t^{n+1}\right), \theta_{h}^{n}\right\rangle \\
\leq & C \frac{\tau}{\lambda \mu}\left\|b\left(t^{n+1}\right)\right\|_{-1}^{2}+\lambda \mu \tau\left\|\nabla \theta_{h}^{n}\right\|_{0}^{2} .
\end{aligned}
$$

Inserting $A_{1}-A_{4}$ back into (39), adding with (46), and then summing over $n$ from 1 to $N$ lead to (13) by help of discrete Gronwall inequality and the equality $\left\|\widehat{\mathbf{u}}_{h}^{n+1}\right\|_{0}^{2}=\left\|\mathbf{u}_{h}^{n+1}\right\|_{0}^{2}+$ $\left\|\nabla \delta \psi_{h}^{n+1}\right\|_{0}^{2}$. So we finish the proof of Theorem 3 .

\section{Error Estimates}

We prove Theorem 4 which is error estimates for SGUM of Algorithm 1. This proof is carried out through several lemmas. We start to prove this theorem with defining $\left(\mathbf{U}_{h}^{n+1}, P_{h}^{n+1}\right):=\mathbb{S}_{h}\left(\mathbf{u}\left(t^{n+1}\right), p\left(t^{n+1}\right)\right) \in \mathbb{V}_{h} \times \mathbb{P}_{h}$ to be 
the Stokes projection of the true solution at time $t^{n+1}$. It means that $\left(\mathbf{U}_{h}^{n+1}, P_{h}^{n+1}\right) \in \mathbb{V}_{h} \times \mathbb{P}_{h}$ is the solution of

$$
\begin{aligned}
& \left\langle\nabla\left(\mathbf{u}\left(t^{n+1}\right)-\mathbf{U}_{h}^{n+1}\right), \nabla \mathbf{w}_{h}\right\rangle \\
& +\left\langle\nabla\left(p\left(t^{n+1}\right)-P_{h}^{n+1}\right), \mathbf{w}_{h}\right\rangle=0, \\
& \forall \mathbf{w}_{h} \in \mathbb{V}_{h}, \\
& \left\langle\nabla \cdot \mathbf{U}_{h}^{n+1}, \phi_{h}\right\rangle=0, \quad \phi_{h} \in \mathbb{P}_{h} .
\end{aligned}
$$

We also define $\Theta_{h}^{n+1} \in \mathbb{T}_{h}$ as the solution of

$$
\left\langle\nabla \Theta_{h}^{n+1}, \nabla \phi_{h}\right\rangle=\left\langle\nabla \theta\left(t^{n+1}\right), \nabla \phi_{h}\right\rangle, \quad \forall \phi_{h} \in \mathbb{T}_{h} .
$$

And we denote notations $\mathbf{G}^{n+1}:=\mathbf{u}\left(t^{n+1}\right)-\mathbf{U}_{h}^{n+1}$,

$$
\begin{aligned}
g^{n+1} & :=p\left(t^{n+1}\right)-P_{h}^{n+1}, \\
\eta^{n+1} & :=\theta\left(t^{n+1}\right)-\Theta_{h}^{n+1} .
\end{aligned}
$$

From Lemma 7, we can deduce

$$
\begin{gathered}
\left\|\mathbf{G}^{n+1}\right\|_{0}^{2}+h^{2}\left\|\mathbf{G}^{n+1}\right\|_{1}^{2}+h^{2}\left\|g^{n+1}\right\|_{0}^{2} \\
\leq C h^{4}\left(\left\|\mathbf{u}\left(t^{n+1}\right)\right\|_{2}^{2}+\left\|p\left(t^{n+1}\right)\right\|_{1}^{2}\right), \\
\left\|\delta \mathbf{G}^{n+1}\right\|_{0}^{2}+h^{2}\left\|\delta \mathbf{G}^{n+1}\right\|_{1}^{2}+h^{2}\left\|\delta g^{n+1}\right\|_{0}^{2} \\
\leq C \tau h^{4} \int_{t^{n}}^{t^{n+1}}\left(\left\|\mathbf{u}_{t}(t)\right\|_{2}^{2}+\left\|p_{t}(t)\right\|_{1}^{2}\right) d t .
\end{gathered}
$$

In conjunction with the definition of $|\|\cdot\||$ in (26), we can derive

$$
\left\|\left|\mathbf{G}^{n+1}\right|\right\| \leq C
$$

We now carry out error evaluation by comparing (65) with (6)-(10) and then by comparing (82) and (11). We derive strong estimates of order 1 , and this result is instrumental in proving weak estimates of order 2 for the errors

$$
\begin{aligned}
\mathbf{E}_{h}^{n+1} & :=\mathbf{U}_{h}^{n+1}-\mathbf{u}_{h}^{n+1}, \\
\widehat{\mathbf{E}}_{h}^{n+1} & :=\mathbf{U}_{h}^{n+1}-\widehat{\mathbf{u}}_{h}^{n+1}, \\
e_{h}^{n+1} & :=P_{h}^{n+1}-p_{h}^{n+1}, \\
\vartheta_{h}^{n+1} & :=\Theta_{h}^{n+1}-\theta_{h}^{n+1} .
\end{aligned}
$$

Then, in conjunction with (50), we can readily get the same accuracy for the errors

$$
\begin{aligned}
\mathbf{E}^{n+1} & :=\mathbf{u}\left(t^{n+1}\right)-\mathbf{u}_{h}^{n+1}, \\
\widehat{\mathbf{E}}^{n+1} & :=\mathbf{u}\left(t^{n+1}\right)-\widehat{\mathbf{u}}_{h}^{n+1}, \\
e^{n+1} & :=p\left(t^{n+1}\right)-p_{h}^{n+1}, \\
\vartheta^{n+1} & :=\theta\left(t^{n+1}\right)-\theta_{h}^{n+1} .
\end{aligned}
$$

Additionally, we denote

$$
\varepsilon_{h}^{n+1}:=P_{h}^{n+1}+\frac{3 \psi_{h}^{n+1}}{2 \tau} .
$$

We readily obtain the following properties:

$$
\begin{aligned}
\mathbf{G}^{n+1} & =\mathbf{E}^{n+1}-\mathbf{E}_{h}^{n+1}=\widehat{\mathbf{E}}^{n+1}-\widehat{\mathbf{E}}_{h}^{n+1}, \\
& \widehat{\mathbf{E}}_{h}^{n+1}=\mathbf{E}_{h}^{n+1}+\nabla \delta \psi_{h}^{n+1}, \\
\widehat{\mathbf{E}}^{n+1}= & \widehat{\mathbf{E}}_{h}^{n+1}=\mathbf{G}^{n+1}=\mathbf{0}, \quad \text { on } \partial \Omega .
\end{aligned}
$$

Moreover, from (12),

$$
\begin{aligned}
\left\langle\mathbf{E}_{h}^{n+1}, \nabla \phi_{h}\right\rangle & =\left\langle\mathbf{E}^{n+1}, \nabla \phi_{h}\right\rangle \\
& =\left\langle\mathbf{G}^{n+1}, \nabla \phi_{h}\right\rangle \\
& =0, \quad \forall \phi_{h} \in \mathbb{P}_{h} .
\end{aligned}
$$

Whence we deduce crucial orthogonality properties:

$$
\left\|\widehat{\mathbf{E}}_{h}^{n+1}\right\|_{0}^{2}=\left\|\mathbf{E}_{h}^{n+1}\right\|_{0}^{2}+\left\|\nabla \delta \psi_{h}^{n+1}\right\|_{0}^{2} .
$$

We also point out that, owing to Lemma $6, q_{h}^{n+1} \in \mathbb{P}_{h}$ defined in (9) satisfies

$$
\left\|q_{h}^{n+1}-q_{h}^{n}\right\|_{0} \leq\left\|\nabla \widehat{\mathbf{E}}^{n+1}\right\|_{0}
$$

In conjunction with $\eta^{n+1}=\theta\left(t^{n+1}\right)-\Theta_{h}^{n+1}=9^{n+1}-\vartheta_{h}^{n+1}$, Assumption 5 leads to

$$
\begin{gathered}
\left\|\eta^{n+1}\right\|_{0}^{2}+h^{2}\left\|\eta^{n+1}\right\|_{1} \leq C h^{4}\left\|\theta\left(t^{n+1}\right)\right\|_{2}^{2}, \\
\left\|\delta \eta^{n+1}\right\|_{0}^{2}+h^{2}\left\|\delta \eta^{n+1}\right\|_{1}^{2} \leq C \tau h^{4} \int_{t^{n}}^{t^{n+1}}\left\|\theta_{t}\right\|_{2} d t .
\end{gathered}
$$

We now estimate the first-order accuracy for velocity and temperature in Lemma 13 and then the 2nd-order accuracy for time derivative of velocity and temperature in Lemma 15. The result of Lemma 13 is instrumental to treat the convection term in proof of Lemma 15. We will use Lemmas 13 and 15 to prove optimal error accuracy in Lemma 17. Finally, we will prove pressure error estimate in Lemma 18.

Lemma 13 (reduced rate of convergence for velocity and temperature). Suppose the exact solution of (1) is smooth enough. 
If Assumptions 3-5 hold, then the velocity and temperature error functions satisfy

$$
\begin{aligned}
& \left\|\widehat{\mathbf{E}}_{h}^{N+1}\right\|_{0}^{2}+\left\|\mathbf{E}_{h}^{N+1}\right\|_{0}^{2}+\left\|2 \mathbf{E}_{h}^{N+1}-\mathbf{E}_{h}^{N}\right\|_{0}^{2} \\
& \quad+2 \mu \tau\left\|q_{h}^{N+1}\right\|_{0}^{2}+\frac{4 \tau^{2}}{3}\left\|\nabla \varepsilon_{h}^{N+1}\right\|_{0}^{2} \\
& \quad+\left\|\vartheta_{h}^{N+1}\right\|_{0}^{2}+\left\|2 \vartheta_{h}^{N+1}-\vartheta_{h}^{N}\right\|_{0}^{2} \\
& \quad+\mu \tau \sum_{n=1}^{N}\left(\left\|\nabla \widehat{\mathbf{E}}_{h}^{n+1}\right\|_{0}^{2}+\lambda\left\|\nabla \vartheta_{h}^{n+1}\right\|_{0}^{2}\right) \\
& \quad+\sum_{n=1}^{N}\left(\left\|\delta \delta \mathbf{E}_{h}^{n+1}\right\|_{0}^{2}+\left\|\nabla \delta \psi_{h}^{n+1}\right\|_{0}^{2}+\left\|\delta \delta \vartheta_{h}^{n+1}\right\|_{0}^{2}\right) \\
& \leq C\left(\tau^{2}+h^{2}\right) .
\end{aligned}
$$

Proof. We resort to the Taylor theorem to write (1) as follows:

$$
\begin{aligned}
& \frac{3 \mathbf{u}\left(t^{n+1}\right)-4 \mathbf{u}\left(t^{n}\right)+\mathbf{u}\left(t^{n-1}\right)}{2 \tau} \\
& +\left(\mathbf{u}\left(t^{n+1}\right) \cdot \nabla\right) \mathbf{u}\left(t^{n+1}\right)+\nabla p\left(t^{n+1}\right) \\
& \quad-\mu \Delta \mathbf{u}\left(t^{n+1}\right)+\kappa \mu^{2} \mathbf{g} \theta\left(t^{n+1}\right) \\
& =\mathbf{R}^{n+1}+\mathbf{f}\left(t^{n+1}\right), \\
& \frac{3 \theta\left(t^{n+1}\right)-4 \theta\left(t^{n}\right)+\theta\left(t^{n-1}\right)}{2 \tau} \\
& \quad+\mathbf{u}\left(t^{n+1}\right) \cdot \nabla \theta\left(t^{n+1}\right)-\lambda \mu \Delta \theta\left(t^{n+1}\right) \\
& =Q^{n+1}+b\left(t^{n+1}\right),
\end{aligned}
$$

where

$$
\begin{aligned}
& \mathbf{R}^{n+1}:=\left(\frac{1}{\tau}\right) \int_{t^{n}}^{t^{n+1}} \mathbf{u}_{t t t}(s)\left(s-t^{n}\right)^{2} d s \\
&-\left(\frac{1}{4 \tau}\right) \int_{t^{n-1}}^{t^{n+1}} \mathbf{u}_{t t t}(s)\left(t^{n-1}-s\right)^{2} d s, \\
& Q^{n+1}:=\left(\frac{1}{\tau}\right) \int_{t^{n}}^{t^{n+1}} \theta_{t t t}(s)\left(s-t^{n}\right)^{2} d s \\
&-\left(\frac{1}{4 \tau}\right) \int_{t^{n-1}}^{t^{n+1}} \theta_{t t t}(s)\left(t^{n-1}-s\right)^{2} d s
\end{aligned}
$$

are the truncation errors. In conjunction with the definition of the Stokes projection $\left\{\mathbf{U}_{h}^{n+1}, P_{h}^{n+1}\right\}$, we readily get a weak formulation for (62), $\forall \mathbf{w}_{h} \in \mathbb{V}_{h}$ :

$$
\begin{aligned}
\frac{1}{2 \tau}\langle & \left.3 \mathbf{u}\left(t^{n+1}\right)-4 \mathbf{u}\left(t^{n}\right)+\mathbf{u}\left(t^{n-1}\right), \mathbf{w}_{h}\right\rangle \\
& +\mathcal{N}\left(\mathbf{u}\left(t^{n+1}\right), \mathbf{u}\left(t^{n+1}\right), \mathbf{w}_{h}\right)+\left\langle\nabla P_{h}^{n+1}, \mathbf{w}_{h}\right\rangle \\
& +\mu\left\langle\nabla \mathbf{U}_{h}^{n+1}, \nabla \mathbf{w}_{h}\right\rangle+\kappa \mu^{2}\left\langle\mathbf{g} \theta\left(t^{n+1}\right), \mathbf{w}_{h}\right\rangle \\
= & \left\langle\mathbf{R}^{n+1}, \mathbf{w}_{h}\right\rangle+\left\langle\mathbf{f}\left(t^{n+1}\right), \mathbf{w}_{h}\right\rangle .
\end{aligned}
$$

We replace the pressure $p_{h}^{n}$ term in (6) with (10) and then subtract it from (65) to obtain

$$
\begin{aligned}
\frac{1}{2 \tau}\langle & \left.\widehat{\mathbf{E}}^{n+1}-4 \mathbf{E}^{n}+\mathbf{E}^{n-1}, \mathbf{w}_{h}\right\rangle \\
& +\mu \tau\left\langle\nabla \widehat{\mathbf{E}}_{h}^{n+1}, \nabla \mathbf{w}_{h}\right\rangle \\
= & -\left\langle\nabla\left(\delta P_{h}^{n+1}+\varepsilon_{h}^{n}\right), \mathbf{w}_{h}\right\rangle+\mu\left\langle\nabla q_{h}^{n}, \mathbf{w}_{h}\right\rangle \\
& +\mathcal{N}\left(2 \mathbf{u}_{h}^{n}-\mathbf{u}_{h}^{n-1}, \widehat{\mathbf{u}}_{h}^{n+1}, \mathbf{w}_{h}\right) \\
& -\mathcal{N}\left(\mathbf{u}\left(t^{n+1}\right), \mathbf{u}\left(t^{n+1}\right), \mathbf{w}_{h}\right) \\
& -\kappa \mu^{2}\left\langle\mathbf{g}\left(\theta\left(t^{n+1}\right)-2 \theta_{h}^{n}+\theta_{h}^{n-1}\right), \mathbf{w}_{h}\right\rangle \\
& +\left\langle\mathbf{R}^{n+1}, \mathbf{w}_{h}\right\rangle .
\end{aligned}
$$

Choosing $\mathbf{w}_{h}=4 \tau \widehat{\mathbf{E}}_{h}^{n+1}=4 \tau\left(\widehat{\mathbf{E}}^{n+1}-\mathbf{G}^{n+1}\right)=4 \tau\left(\mathbf{E}_{h}^{n+1}+\right.$ $\left.\nabla \delta \psi_{h}^{n+1}\right)$ in (66) and using (58) and (35) yield

$$
\begin{aligned}
\delta\left\|\mathbf{E}_{h}^{n+1}\right\|_{0}^{2}+\delta\left\|2 \mathbf{E}_{h}^{n+1}-\mathbf{E}_{h}^{n}\right\|_{0}^{2} \\
+\left\|\delta \delta \mathbf{E}_{h}^{n+1}\right\|_{0}^{2}+6\left\|\nabla \delta \psi_{h}^{n+1}\right\|_{0}^{2} \\
\quad+4 \mu \tau\left\|\nabla \widehat{\mathbf{E}}_{h}^{n+1}\right\|_{0}^{2} \\
=\sum_{n=1}^{7} A_{i},
\end{aligned}
$$

where

$$
\begin{gathered}
A_{1}:=-4 \tau \mathcal{N}\left(\mathbf{u}\left(t^{n+1}\right), \mathbf{u}\left(t^{n+1}\right), \widehat{\mathbf{E}}_{h}^{n+1}\right) \\
+4 \tau \mathcal{N}\left(2 \mathbf{u}_{h}^{n}-\mathbf{u}_{h}^{n-1}, \widehat{\mathbf{u}}_{h}^{n+1}, \widehat{\mathbf{E}}_{h}^{n+1}\right), \\
A_{2}:=-2\left\langle 3 \mathbf{G}^{n+1}-4 \mathbf{G}^{n}+\mathbf{G}^{n-1}, \widehat{\mathbf{E}}_{h}^{n+1}\right\rangle, \\
A_{3}:=-4 \tau\left\langle\nabla \delta P_{h}^{n+1}, \widehat{\mathbf{E}}_{h}^{n+1}\right\rangle, \\
A_{4}:=-4 \tau\left\langle\nabla \varepsilon_{h}^{n}, \widehat{\mathbf{E}}_{h}^{n+1}\right\rangle, \\
A_{5}:=4 \mu \tau\left\langle\nabla q_{h}^{n}, \widehat{\mathbf{E}}_{h}^{n+1}\right\rangle, \\
A_{6}:=4 \tau\left\langle\mathbf{R}^{n+1}, \widehat{\mathbf{E}}_{h}^{n+1}\right\rangle, \\
A_{7}:=-4 \tau \kappa \mu^{2}\left\langle\mathbf{g}\left(\theta\left(t^{n+1}\right)-2 \theta_{h}^{n}+\theta_{h}^{n-1}\right), \widehat{\mathbf{E}}_{h}^{n+1}\right\rangle .
\end{gathered}
$$

We now estimate all the terms from $A_{1}$ to $A_{7}$, respectively. To tackle $A_{1}$, we first add and subtract $2 \mathbf{u}\left(t^{n}\right)-\mathbf{u}\left(t^{n-1}\right)$ to obtain

$$
\begin{aligned}
A_{1}= & -4 \tau \mathscr{N}\left(\delta \delta \mathbf{u}\left(t^{n+1}\right), \mathbf{u}\left(t^{n+1}\right), \widehat{\mathbf{E}}_{h}^{n+1}\right) \\
& -4 \tau \mathcal{N}\left(2 \mathbf{u}_{h}^{n}-\mathbf{u}_{h}^{n-1}, \widehat{\mathbf{E}}^{n+1}, \widehat{\mathbf{E}}_{h}^{n+1}\right) \\
& -4 \tau \mathcal{N}\left(2 \mathbf{E}^{n}-\mathbf{E}^{n-1}, \mathbf{u}\left(t^{n+1}\right), \widehat{\mathbf{E}}_{h}^{n+1}\right) .
\end{aligned}
$$

Because of $\mathcal{N}\left(2 \mathbf{u}_{h}^{n}-\mathbf{u}_{h}^{n-1}, \widehat{\mathbf{E}}_{h}^{n+1}, \widehat{\mathbf{E}}_{h}^{n+1}\right)=0$, which comes from (30), the second term of $A_{1}$ can be replaced by

$$
4 \tau \mathcal{N}\left(2 \mathbf{E}^{n}-\mathbf{E}^{n-1}-2 \mathbf{u}\left(t^{n}\right)+\mathbf{u}\left(t^{n-1}\right), \mathbf{G}^{n+1}, \widehat{\mathbf{E}}_{h}^{n+1}\right) .
$$


If we apply Lemma 10, then we can readily obtain

$$
\begin{aligned}
A_{1} \leq C & \tau\left(\left\|\delta \delta \mathbf{u}\left(t^{n+1}\right)\right\|_{0}\left\|\mathbf{u}\left(t^{n+1}\right)\right\|_{2}\right. \\
+ & \left.\left\|2 \mathbf{E}^{n}-\mathbf{E}^{n-1}\right\|_{0}\left\|\mathbf{u}\left(t^{n+1}\right)\right\|_{2}\right)\left\|\widehat{\mathbf{E}}_{h}^{n+1}\right\|_{1} \\
+C & \tau\left(\left\|2 \mathbf{E}^{n}-\mathbf{E}^{n-1}\right\|_{0}\left\|\left|\mathbf{G}^{n+1}\right|\right\|\right. \\
& \left.+\left\|2 \mathbf{u}\left(t^{n}\right)-\mathbf{u}\left(t^{n-1}\right)\right\|_{2}\left\|\mathbf{G}^{n+1}\right\|_{0}\right)\left\|\widehat{\mathbf{E}}_{h}^{n+1}\right\|_{1} .
\end{aligned}
$$

Since we have $\left\|\mathbf{u}\left(t^{n+1}\right)\right\|_{2}+\left\|\left|\mathbf{G}^{n+1}\right|\right\| \leq M$ according to (52), we arrive at

$$
\begin{aligned}
A_{1} \leq & C \tau\left(\left\|2 \mathbf{E}_{h}^{n}-\mathbf{E}_{h}^{n-1}\right\|_{0}^{2}\right. \\
& \left.+\left\|2 \mathbf{G}^{n}-\mathbf{G}^{n-1}\right\|_{0}^{2}+\left\|\mathrm{G}^{n+1}\right\|_{0}^{2}\right) \\
& +\mu \tau\left\|\nabla \widehat{\mathbf{E}}_{h}^{n+1}\right\|_{0}^{2}+\frac{C \tau^{4}}{\mu} \\
& \times \int_{t^{n-1}}^{t^{n+1}}\left\|\mathbf{u}_{t t t}(t)\right\|_{0}^{2} d t .
\end{aligned}
$$

In light of (51) and (58), $A_{2}$ becomes

$$
\begin{aligned}
A_{2} \leq & C \tau\left\|\mathbf{E}_{h}^{n+1}\right\|_{0}^{2}+\frac{1}{4}\left\|\nabla \delta \psi_{h}^{n+1}\right\|_{0}^{2} \\
& +C h^{4} \int_{t^{n-1}}^{t^{n+1}}\left(\left\|\mathbf{u}_{t}(t)\right\|_{2}^{2}+\left\|p_{t}(t)\right\|_{1}^{2}\right) d t .
\end{aligned}
$$

In order to estimate $A_{3}$ and $A_{4}$, we note that the cancellation law (12) gives $\left\langle\nabla \varepsilon_{h}^{n}, \mathbf{E}_{h}^{n+1}\right\rangle=0$. Then $\widehat{\mathbf{E}}_{h}^{n+1}=\mathbf{E}_{h}^{n+1}+\nabla \delta \psi_{h}^{n+1}$ yields

$$
\begin{aligned}
A_{3}= & -4 \tau\left\langle\nabla \delta P_{h}^{n+1}, \nabla \delta \psi_{h}^{n+1}\right\rangle \\
\leq & \frac{1}{4}\left\|\nabla \delta \psi_{h}^{n+1}\right\|_{0}^{2}+C \tau^{3} \\
& \times \int_{t^{n}}^{t^{n+1}}\left(\left\|\mathbf{u}_{t}(t)\right\|_{2}^{2}+\left\|p_{t}(t)\right\|_{1}^{2}\right) d t
\end{aligned}
$$

In conjunction with the definition $\varepsilon_{h}^{n+1}=P_{h}^{n+1}+3 \psi_{h}^{n+1} / 2 \tau$, $A_{4}$ can be evaluated by

$$
\begin{aligned}
A_{4}= & -4 \tau\left\langle\nabla \varepsilon_{h}^{n}, \nabla \delta \psi_{h}^{n+1}\right\rangle \\
= & -\frac{8 \tau^{2}}{3}\left\langle\nabla \varepsilon_{h}^{n}, \nabla\left(\delta \varepsilon_{h}^{n+1}-\delta P_{h}^{n+1}\right)\right\rangle \\
\leq & -\frac{4 \tau^{2}}{3}\left(\left\|\nabla \varepsilon_{h}^{n+1}\right\|_{0}^{2}-\left\|\nabla \varepsilon_{h}^{n}\right\|_{0}^{2}-\left\|\nabla \delta \varepsilon_{h}^{n+1}\right\|_{0}^{2}\right) \\
& +C \tau^{3}\left\|\nabla \varepsilon_{h}^{n}\right\|_{0}^{2}+C \tau\left\|\nabla \delta P_{h}^{n+1}\right\|_{0}^{2} .
\end{aligned}
$$

If we now apply inequality $(a+b)^{2} \leq 4 a^{2}+(4 / 3) b^{2}$, then we can get

$$
\begin{aligned}
\frac{4 \tau^{2}}{3} \| & \nabla \delta \varepsilon_{h}^{n+1} \|_{0}^{2} \\
& =\frac{4 \tau^{2}}{3}\left\|\nabla \delta P_{h}^{n+1}+\frac{3}{2 \tau} \nabla \delta \psi_{h}^{n+1}\right\|_{0}^{2} \\
\leq & C \tau^{2}\left\|\nabla \delta P_{h}^{n+1}\right\|_{0}^{2}+4\left\|\nabla \delta \psi_{h}^{n+1}\right\|_{0}^{2} .
\end{aligned}
$$

So we arrive at

$$
\begin{aligned}
A_{4} \leq & -\frac{4 \tau^{2}}{3}\left(\left\|\nabla \varepsilon_{h}^{n+1}\right\|_{0}^{2}-\left\|\nabla \varepsilon_{h}^{n}\right\|_{0}^{2}\right) \\
& +C \tau^{3}\left\|\nabla \varepsilon_{h}^{n}\right\|_{0}^{2}+4\left\|\nabla \delta \psi_{h}^{n+1}\right\|_{0}^{2} \\
& +C \tau^{2} \int_{t^{n}}^{t^{n+1}}\left(\left\|\mathbf{u}_{t}(t)\right\|_{2}^{2}+\left\|p_{t}(t)\right\|_{1}^{2}\right) d t .
\end{aligned}
$$

In light of $\nabla \cdot \mathbf{u}\left(t^{n+1}\right)=0$ and (37), (9) and (59) yield

$$
\begin{aligned}
A_{5}= & 4 \mu \tau\left\langle q_{h}^{n}, \nabla \cdot \widehat{\mathbf{u}}_{h}^{n+1}\right\rangle \\
= & -4 \mu \tau\left\langle q_{h}^{n}, \delta q_{h}^{n+1}\right\rangle \\
= & -2 \mu \tau\left(\left\|q_{h}^{n+1}\right\|_{0}^{2}-\left\|q_{h}^{n}\right\|_{0}^{2}-\left\|\delta q_{h}^{n+1}\right\|_{0}^{2}\right) \\
\leq & -2 \mu \tau\left(\left\|q_{h}^{n+1}\right\|_{0}^{2}-\left\|q_{h}^{n}\right\|_{0}^{2}\right) \\
& +2 \mu \tau\left\|\nabla \widehat{\mathbf{E}}_{h}^{n+1}\right\|_{0}^{2} .
\end{aligned}
$$

Also we readily get

$$
\begin{aligned}
A_{6} \leq & C \tau\left\|\mathbf{E}_{h}^{n+1}\right\|_{0}^{2}+\frac{1}{4}\left\|\nabla \delta \psi_{h}^{n+1}\right\|_{0}^{2} \\
& +C \tau^{4} \int_{t_{n-1}}^{t^{n+1}}\left\|\mathbf{u}_{t t t}(t)\right\|_{0}^{2} d t .
\end{aligned}
$$

The Hölder inequality and (60) yield

$$
\begin{aligned}
A_{7}= & -4 \kappa \mu^{2} \tau\left\langle\mathbf{g}\left(\delta \delta \theta\left(t^{n+1}\right)-2 \vartheta^{n}+\vartheta^{n-1}\right), \widehat{\mathbf{E}}_{h}^{n+1}\right\rangle \\
\leq & C \kappa^{2} \mu^{4} \tau\left(\left\|2 \vartheta_{h}^{n}-\vartheta_{h}^{n-1}\right\|_{0}^{2}+\left\|2 \eta^{n}-\eta^{n-1}\right\|_{0}^{2}\right) \\
& +\tau\left\|\mathbf{E}_{h}^{n+1}\right\|_{0}^{2}+\frac{1}{4}\left\|\nabla \delta \psi_{h}^{n+1}\right\|_{0}^{2} \\
& +C \tau^{4} \int_{t^{n-1}}^{t^{n}+1}\left\|\theta_{t t}(t)\right\|_{0}^{2} d t .
\end{aligned}
$$


Inserting the above estimates into (67) gives

$$
\begin{aligned}
\delta\left\|\mathbf{E}_{h}^{n+1}\right\|_{0}^{2}+\delta\left\|2 \mathbf{E}_{h}^{n+1}-\mathbf{E}_{h}^{n}\right\|_{0}^{2} & \\
+ & \left\|\delta \delta \mathbf{E}_{h}^{n+1}\right\|_{0}^{2}+\left\|\nabla \delta \psi_{h}^{n+1}\right\|_{0}^{2}+\mu \tau\left\|\nabla \widehat{\mathbf{E}}_{h}^{n+1}\right\|_{0}^{2} \\
& +\frac{4 \tau^{2}}{3}\left\|\nabla \varepsilon_{h}^{n+1}\right\|_{0}^{2}+2 \mu \tau\left\|q_{h}^{n+1}\right\|_{0}^{2} \\
\leq & \frac{4 \tau^{2}}{3}\left\|\nabla \varepsilon_{h}^{n}\right\|_{0}^{2}+C \tau^{3}\left\|\nabla \varepsilon_{h}^{n}\right\|_{0}^{2}+2 \mu \tau\left\|q_{h}^{n}\right\|_{0}^{2} \\
& +C \tau\left(\left\|\mathbf{E}_{h}^{n+1}\right\|_{0}^{2}+\left\|2 \mathbf{E}_{h}^{n}-\mathbf{E}_{h}^{n-1}\right\|_{0}^{2}\right. \\
& \left.+\left\|2 \mathbf{G}^{n}-\mathbf{G}^{n-1}\right\|_{0}^{2}+\left\|\mathbf{G}^{n+1}\right\|_{0}^{2}\right) \\
& +C \kappa^{2} \mu^{4} \tau\left\|^{n}-\eta^{n-1}\right\|_{0}^{2} \\
& +C \tau^{4} \int_{t^{n-1}}^{t^{n}+1}\left(\left\|\mathbf{u}_{t t t}(t)\right\|_{0}^{2}+\left\|\theta_{t t}(t)\right\|_{0}^{2}\right) d t \\
& +C \kappa^{2} \mu^{4} \tau\left\|2 \vartheta_{h}^{n}-\vartheta_{h}^{n-1}\right\|_{0}^{2} \\
& +C\left(\tau^{2}+h^{4}\right) \int_{t^{n-1}}^{t^{n+1}}\left(\left\|\mathbf{u}_{t}(t)\right\|_{2}^{2}+\left\|p_{t}(t)\right\|_{1}^{2}\right) d t .
\end{aligned}
$$

On the other hand, the definition (48) of $\Theta_{h}^{n+1} \in \mathbb{T}_{h}$ leads to a weak formula of (63) as, for all $\phi_{h} \in \mathbb{T}_{h}$,

$$
\begin{aligned}
\frac{1}{2 \tau}\langle & \left.3 \theta\left(t^{n+1}\right)-4 \theta\left(t^{n}\right)+\theta\left(t^{n-1}\right), \phi_{h}\right\rangle \\
& +\mathcal{N}\left(\mathbf{u}\left(t^{n+1}\right), \theta\left(t^{n+1}\right), \phi_{h}\right) \\
& +\lambda \mu\left\langle\nabla \Theta_{h}^{n+1}, \nabla \phi_{h}\right\rangle \\
= & \left\langle Q^{n+1}+b\left(t^{n+1}\right), \phi_{h}\right\rangle .
\end{aligned}
$$

We now subtract (11) from (82) to derive

$$
\begin{aligned}
\frac{1}{2 \tau}\langle & \left.3 \vartheta^{n+1}-4 \vartheta^{n}+\vartheta^{n-1}, \phi_{h}\right\rangle \\
& +\mathcal{N}\left(\mathbf{u}\left(t^{n+1}\right), \theta\left(t^{n+1}\right), \phi_{h}\right) \\
& -\mathcal{N}\left(\mathbf{u}_{h}^{n+1}, \theta_{h}^{n+1}, \phi_{h}\right) \\
& +\lambda \mu\left\langle\nabla \vartheta_{h}^{n+1}, \nabla \phi_{h}\right\rangle \\
= & \left\langle Q^{n+1}, \phi_{h}\right\rangle .
\end{aligned}
$$

Choosing $\phi_{h}=4 \tau \vartheta_{h}^{n+1}=4 \tau\left(\vartheta^{n+1}-\eta^{n+1}\right)$ yields

$$
\begin{aligned}
\delta\left\|\vartheta_{h}^{n+1}\right\|_{0}^{2}+\delta\left\|2 \vartheta_{h}^{n+1}-\vartheta_{h}^{n}\right\|_{0}^{2} \\
\quad+\left\|\delta \delta \vartheta_{h}^{n+1}\right\|_{0}^{2}+4 \lambda \mu \tau\left\|\nabla \vartheta_{h}^{n+1}\right\|_{0}^{2} \\
=A_{8}+A_{9},
\end{aligned}
$$

where

$$
\begin{aligned}
A_{8}:= & -4 \tau \mathcal{N}\left(\mathbf{u}\left(t^{n+1}\right), \theta\left(t^{n+1}\right), \vartheta_{h}^{n+1}\right) \\
& +4 \tau \mathcal{N}\left(\mathbf{u}_{h}^{n+1}, \theta_{h}^{n+1}, \vartheta_{h}^{n+1}\right), \\
A_{9}:= & -2\left\langle 3 \eta^{n+1}-4 \eta^{n}+\eta^{n-1}, \vartheta_{h}^{n+1}\right\rangle \\
& +4 \tau\left\langle Q^{n+1}, \vartheta_{h}^{n+1}\right\rangle .
\end{aligned}
$$

To estimate $A_{8}$, we note $\mathcal{N}\left(\mathbf{E}^{n+1}-\mathbf{u}\left(t^{n+1}\right), \vartheta_{h}^{n+1}, \vartheta_{h}^{n+1}\right)=0$ which comes from (30), then $\left\|\mathbf{E}^{n+1}\right\|_{\mathbf{L}^{3}(\Omega)} \leq C h^{-1 / 2}\left\|\mathbf{E}^{n+1}\right\|_{0}$ and $\left\|\eta^{n+1}\right\|_{1} \leq \operatorname{Ch}\left\|\theta\left(t^{n+1}\right)\right\|_{2}$ yield

$$
\begin{aligned}
A_{8}= & -4 \tau \mathcal{N}\left(\mathbf{E}^{n+1}, \theta\left(t^{n+1}\right), \vartheta_{h}^{n+1}\right) \\
& +4 \tau \mathcal{N}\left(\mathbf{E}^{n+1}-\mathbf{u}\left(t^{n+1}\right), \eta^{n+1}, \vartheta_{h}^{n+1}\right) \\
\leq & C \tau\left\|\mathbf{E}^{n+1}\right\|_{0}\left\|\theta\left(t^{n+1}\right)\right\|\left\|_{2}\right\| \vartheta_{h}^{n+1} \|_{1} \\
& +C \tau\left(\left\|\mathbf{E}^{n+1}\right\|_{\mathbf{L}^{3}(\Omega)}\left\|\eta^{n+1}\right\|_{1}\right. \\
& \left.\quad+\left\|\mathbf{u}\left(t^{n+1}\right)\right\|_{2}\left\|\eta^{n+1}\right\|_{0}\right)\left\|\vartheta_{h}^{n+1}\right\|_{1} \\
\leq & \frac{C \tau}{\lambda \mu}\left(\left\|\mathbf{E}^{n+1}\right\|_{0}^{2}+\left\|\eta^{n+1}\right\|_{0}^{2}\right)+\lambda \mu \tau\left\|\nabla \vartheta_{h}^{n+1}\right\|_{0}^{2} .
\end{aligned}
$$

We use $\left\|\delta \eta^{n+1}\right\|_{0}^{2} \leq C \tau h^{4} \int_{t^{n}}^{t^{n+1}}\left\|\theta_{t}(t)\right\|_{2}^{2} d t$ which is (60) to attack $A_{9}$ :

$$
\begin{aligned}
A_{9} \leq & C\left\|3 \eta^{n+1}-4 \eta^{n}+\eta^{n-1}\right\|_{0}\left\|\vartheta_{h}^{n+1}\right\|_{0} \\
& +C \tau\left\|Q^{n+1}\right\|_{0}\left\|\vartheta_{h}^{n+1}\right\|_{0} \\
\leq & C \tau\left\|\vartheta_{h}^{n+1}\right\|_{0}^{2}+C h^{4} \int_{t^{n-1}}^{t^{n+1}}\left\|\theta_{t}(t)\right\|_{2}^{2} d t \\
& +C \tau^{4} \int_{t^{n-1}}^{t^{n+1}}\left\|\theta_{t t t}(t)\right\|_{0}^{2} d t .
\end{aligned}
$$

Inserting the above estimates into (84) gives

$$
\begin{aligned}
\delta\left\|\vartheta_{h}^{n+1}\right\|_{0}^{2}+\delta\left\|2 \vartheta_{h}^{n+1}-\vartheta_{h}^{n}\right\|_{0}^{2} & \\
& +\left\|\delta \delta \vartheta_{h}^{n+1}\right\|_{0}^{2}+3 \lambda \mu \tau\left\|\nabla \vartheta_{h}^{n+1}\right\|_{0}^{2} \\
\leq & C \tau\left\|\vartheta_{h}^{n+1}\right\|_{0}^{2}+\frac{C \tau}{\lambda \mu}\left(\left\|\mathbf{E}^{n+1}\right\|_{0}^{2}+\left\|\eta^{n+1}\right\|_{0}^{2}\right) \\
& +C\left(h^{4}+\tau^{4}\right) \int_{t^{n-1}}^{t^{n+1}}\left(\left\|\theta_{t t t}(t)\right\|_{0}^{2}+\left\|\theta_{t}(t)\right\|_{2}^{2}\right) d t
\end{aligned}
$$


Adding 2 inequalities (81) and (88) and summing over $n$ from 1 to $N$ imply

$$
\begin{aligned}
& \left\|\mathbf{E}_{h}^{N+1}\right\|_{0}^{2}+\left\|2 \mathbf{E}_{h}^{N+1}-\mathbf{E}_{h}^{N}\right\|_{0}^{2} \\
& +\left\|\vartheta_{h}^{N+1}\right\|_{0}^{2}+\left\|2 \vartheta_{h}^{N+1}-\vartheta_{h}^{N}\right\|_{0}^{2} \\
& +2 \mu \tau\left\|q_{h}^{n+1}\right\|_{0}^{2}+\frac{4 \tau^{2}}{3}\left\|\nabla \varepsilon_{h}^{n+1}\right\|_{0}^{2} \\
& +\sum_{n=1}^{N}\left(\left\|\delta \delta \mathbf{E}_{h}^{N+1}\right\|_{0}^{2}+\left\|\delta \delta \vartheta_{h}^{n+1}\right\|_{0}^{2}\right. \\
& \left.+\left\|\nabla \delta \psi_{h}^{n+1}\right\|_{0}^{2}\right) \\
& +\mu \tau \sum_{n=1}^{N}\left(\left\|\nabla \widehat{\mathbf{E}}_{h}^{n+1}\right\|_{0}^{2}+3 \lambda\left\|\nabla \vartheta_{h}^{n+1}\right\|_{0}^{2}\right) \\
& \leq\left\|\mathbf{E}_{h}^{1}\right\|_{0}^{2}+\left\|2 \mathbf{E}_{h}^{1}-\mathbf{E}_{h}^{0}\right\|_{0}^{2}+\left\|\vartheta_{h}^{1}\right\|_{0}^{2} \\
& +\left\|2 \vartheta_{h}^{1}-\vartheta_{h}^{0}\right\|_{0}^{2}+\frac{4 \tau^{2}}{3}\left\|\nabla \varepsilon_{h}^{1}\right\|_{0}^{2}+2 \mu \tau\left\|q_{h}^{1}\right\|_{0}^{2} \\
& +\tau \sum_{n=1}^{N}\left(\left\|\mathbf{E}^{n+1}\right\|_{0}^{2}+\left\|\eta^{n+1}\right\|_{0}^{2}\right) \\
& +C \tau \sum_{n=1}^{N}\left(\left\|\vartheta_{h}^{n+1}\right\|_{0}^{2}+\left\|2 \vartheta_{h}^{n}-\vartheta_{h}^{n-1}\right\|_{0}^{2}\right. \\
& \left.+\left\|2 \eta^{n}-\eta^{n-1}\right\|_{0}^{2}\right) \\
& +C \tau^{3}\left\|\nabla \varepsilon_{h}^{n}\right\|_{0}^{2}+C\left(h^{4}+\tau^{4}\right) \\
& \times \int_{t^{n-1}}^{t^{n+1}}\left(\left\|\mathbf{u}_{t t t}(t)\right\|_{0}^{2}+\left\|\theta_{t t t}(t)\right\|_{0}^{2}\right. \\
& \left.+\left\|\theta_{t}(t)\right\|_{2}^{2}\right) d t \\
& +C\left(\tau^{2}+h^{4}\right) \int_{t^{n-1}}^{t^{n+1}}\left(\left\|\mathbf{u}_{t}(t)\right\|_{2}^{2}+\left\|p_{t}(t)\right\|_{1}^{2}\right) d t .
\end{aligned}
$$

Because of $q^{1}=0$, we have $\varepsilon_{h}^{1}=P_{h}^{1}+(3 / 2 \tau) \psi_{h}^{1}=e_{h}^{1}$, and thus, Assumption 4 yields $\left\|\nabla \varepsilon_{h}^{1}\right\|_{0}^{2} \leq C$ and the first 4 terms in the right-hand side can be bound by Assumption 4 and properties $\mathbf{E}^{0}=\mathbf{0}$ and $q^{1}=0$ which are directly deduced from the conditions in Algorithm 1. Moreover, the remaining terms can be treated by the discrete Gronwall lemma. Finally, in conjunction with (58), we conclude the desired result and complete this proof.

Remark 14 (optimal estimation). In order to get optimal accuracy, we must get rid of the terms of $A_{3}$ and $A_{4}$ by applying duality argument in Lemma 17. To do this, we first evaluate the errors for time derivative of velocity and temperature in Lemma 15. Thus, we need to evaluate optimal initial errors for the case $n=1$, and so we have to recompute again (77). We start to rewrite $A_{4}$ as

$$
\begin{aligned}
A_{4}= & -\frac{8 \tau^{2}}{3}\left\langle\nabla \varepsilon_{h}^{1}, \nabla\left(\delta \varepsilon_{h}^{2}-\delta P_{h}^{2}\right)\right\rangle \\
\leq & -\frac{4 \tau^{2}}{3}\left(\left\|\nabla \varepsilon_{h}^{2}\right\|_{0}^{2}-\left\|\nabla \varepsilon_{h}^{1}\right\|_{0}^{2}-\left\|\nabla \delta \varepsilon_{h}^{2}\right\|_{0}^{2}\right) \\
& +C \tau^{2}\left\|\nabla \varepsilon_{h}^{1}\right\|_{0}^{2}+C \tau^{2}\left\|\nabla \delta P_{h}^{n+1}\right\|_{0}^{2} \\
\leq & -\frac{4 \tau^{2}}{3}\left\|\nabla \varepsilon_{h}^{2}\right\|_{0}^{2}+C \tau^{2}\left\|\nabla \varepsilon_{h}^{1}\right\|_{0}^{2} \\
& +4\left\|\nabla \delta \psi_{h}^{2}\right\|_{0}^{2}+C \tau^{3} \\
& \times \int_{t^{0}}^{t^{2}}\left(\left\|p_{t t}(t)\right\|_{1}^{2}+\left\|\mathbf{u}_{t t}(t)\right\|_{2}^{2}\right) d t .
\end{aligned}
$$

In light of Assumption 4, we arrive at

$$
\begin{aligned}
\left\|\widehat{\mathbf{E}}_{h}^{2}\right\|_{0}^{2} & +\left\|\mathbf{E}_{h}^{2}\right\|_{0}^{2}+\left\|2 \mathbf{E}_{h}^{2}-\mathbf{E}_{h}^{1}\right\|_{0}^{2} \\
& +\frac{1}{2}\left\|\delta \delta \mathbf{E}_{h}^{2}\right\|_{0}^{2}+\left\|\nabla \delta \psi_{h}^{2}\right\|_{0}^{2} \\
& +\left\|2 \vartheta_{h}^{2}-\vartheta_{h}^{2}\right\|_{0}^{2}+\left\|\vartheta_{h}^{2}\right\|_{0}^{2} \\
& +\mu \tau\left\|\nabla \widehat{\mathbf{E}}_{h}^{2}\right\|_{0}^{2}+2 \mu \tau\left\|q_{h}^{2}\right\|_{0}^{2} \\
& +\frac{4 \tau^{2}}{3}\left\|\nabla \varepsilon_{h}^{2}\right\|_{0}^{2}+\mu \lambda \tau\left\|\nabla \vartheta_{h}^{2}\right\|_{0}^{2} \\
\leq & C\left(\tau^{4}+h^{4}\right) .
\end{aligned}
$$

We now start to estimate errors for time derivative of velocity.

Lemma 15 (error estimate for time derivative of velocity and temperature). Suppose the exact solution of (1) is smooth enough and $\tau \leq C h$. If Assumptions 3-5 hold, then the time derivative velocity and temperature error functions satisfy

$$
\begin{aligned}
\left\|\delta \mathbf{E}_{h}^{N+1}\right\|_{0}^{2}+\left\|\delta \widehat{\mathbf{E}}_{h}^{N+1}\right\|_{0}^{2} \\
+\left\|2 \delta \mathbf{E}_{h}^{N+1}-\delta \mathbf{E}_{h}^{N}\right\|_{0}^{2} \\
+2 \mu \tau\left\|\delta q_{h}^{N+1}\right\|_{0}^{2}+\left\|\delta \vartheta_{h}^{N+1}\right\|_{0}^{2} \\
+\left\|2 \delta \vartheta_{h}^{N+1}-\delta \vartheta_{h}^{N}\right\|_{0}^{2} \\
+\sum_{n=2}^{N}\left(\left\|\delta \delta \delta \mathbf{E}_{h}^{n+1}\right\|_{0}^{2}+\left\|\nabla \delta \delta \psi_{h}^{n+1}\right\|_{0}^{2}\right. \\
\left.\quad+\left\|\delta \delta \delta \vartheta_{h}^{n+1}\right\|_{0}^{2}\right) \\
+\frac{4 \tau^{2}}{3}\left\|\nabla \delta \varepsilon_{h}^{N+1}\right\|_{0}^{2} \\
+\mu \tau \sum_{n=2}^{N}\left(\left\|\nabla \delta \widehat{\mathbf{E}}_{h}^{n+1}\right\|_{0}^{2}+\lambda\left\|\nabla \delta \vartheta_{h}^{n+1}\right\|_{0}^{2}\right) \\
\leq C\left(\tau^{2}+h^{2}\right)^{2} .
\end{aligned}
$$


Remark 16 (the condition $\tau \leq C h$ ). The assumption $\tau \leq$ $\mathrm{Ch}$ requires to control convection terms which are used at only (100) and (117), so we can omit this condition for the linearized Boussinesq equations. However, this condition cannot be removed for nonlinear equation case, because (99) must be bounded by $h$.

Proof. We subtract two consecutive formulas of (66) and impose $\mathbf{w}_{h}=4 \tau \delta \widehat{\mathbf{E}}_{h}^{n+1}$ to obtain

$$
\begin{aligned}
& \left\|\delta \mathbf{E}_{h}^{n+1}\right\|_{0}^{2}+\left\|2 \delta \mathbf{E}_{h}^{n+1}-\delta \mathbf{E}_{h}^{n}\right\|_{0}^{2} \\
& +\left\|\delta \delta \delta \mathbf{E}_{h}^{n+1}\right\|_{0}^{2}+6\left\|\nabla \delta \delta \psi_{h}^{n+1}\right\|_{0}^{2} \\
& \quad-\left\|\delta \mathbf{E}_{h}^{n}\right\|_{0}^{2}-\left\|2 \delta \mathbf{E}_{h}^{n}-\delta \mathbf{E}_{h}^{n-1}\right\|_{0}^{2} \\
& \quad+4 \mu \tau\left\|\nabla \delta \widehat{\mathbf{E}}_{h}^{n+1}\right\|_{0}^{2} \\
& =\sum_{n=1}^{7} A_{i},
\end{aligned}
$$

where

$$
\begin{aligned}
& A_{1}:= 4 \tau \mathcal{N}\left(\mathbf{u}\left(t^{n}\right), \mathbf{u}\left(t^{n}\right), \delta \widehat{\mathbf{E}}_{h}^{n+1}\right) \\
&-4 \tau \mathcal{N}\left(2 \mathbf{u}_{h}^{n-1}-\mathbf{u}_{h}^{n-2}, \widehat{\mathbf{u}}_{h}^{n}, \delta \widehat{\mathbf{E}}_{h}^{n+1}\right) \\
&-4 \tau \mathcal{N}\left(\mathbf{u}\left(t^{n+1}\right), \mathbf{u}\left(t^{n+1}\right), \delta \widehat{\mathbf{E}}_{h}^{n+1}\right) \\
&+4 \tau \mathcal{N}\left(2 \mathbf{u}_{h}^{n}-\mathbf{u}_{h}^{n-1}, \widehat{\mathbf{u}}_{h}^{n+1}, \delta \widehat{\mathbf{E}}_{h}^{n+1}\right), \\
& A_{2}:=-2\left\langle 3 \delta \mathbf{G}^{n+1}-4 \delta \mathbf{G}^{n}+\delta \mathbf{G}^{n-1}, \delta \widehat{\mathbf{E}}_{h}^{n+1}\right\rangle, \\
& A_{3}:=-4 \tau\left\langle\nabla \delta \delta P_{h}^{n+1}, \delta \widehat{\mathbf{E}}_{h}^{n+1}\right\rangle, \\
& A_{4}:=-4 \tau\left\langle\nabla \delta \varepsilon_{h}^{n}, \delta \widehat{\mathbf{E}}_{h}^{n+1}\right\rangle, \\
& A_{5}:=4 \mu \tau\left\langle\nabla \delta q_{h}^{n}, \delta \widehat{\mathbf{E}}_{h}^{n+1}\right\rangle, \\
& A_{6}:=4 \tau\left\langle\delta \mathbf{R}^{n+1}, \delta \widehat{\mathbf{E}}_{h}^{n+1}\right\rangle, \\
& A_{7}:=4 \kappa \mu^{2} \tau \\
& \times\left\langle\mathbf{g} \delta\left(\theta\left(t^{n+1}\right)-2 \theta^{n}+\theta^{n-1}\right), \delta \widehat{\mathbf{E}}_{h}^{n+1}\right\rangle .
\end{aligned}
$$

We now estimate each term from $A_{1}$ to $A_{7}$ separately. The convection term $A_{1}$ can be rewritten as follows:

$$
\begin{aligned}
A_{1}= & 4 \tau \mathcal{N}\left(\delta \delta \mathbf{u}\left(t^{n}\right), \mathbf{u}\left(t^{n}\right), \delta \widehat{\mathbf{E}}^{n+1}\right) \\
& -4 \tau \mathcal{N}\left(\delta \delta \mathbf{u}\left(t^{n+1}\right), \mathbf{u}\left(t^{n+1}\right), \delta \widehat{\mathbf{E}}^{n+1}\right) \\
& +4 \tau \mathcal{N}\left(2 \mathbf{E}^{n-1}-\mathbf{E}^{n-2}, \mathbf{u}\left(t^{n}\right), \delta \widehat{\mathbf{E}}^{n+1}\right) \\
& -4 \tau \mathcal{N}\left(2 \mathbf{E}^{n}-\mathbf{E}^{n-1}, \mathbf{u}\left(t^{n+1}\right), \delta \widehat{\mathbf{E}}^{n+1}\right) \\
& +4 \tau \mathcal{N}\left(2 \mathbf{u}_{h}^{n-1}-\mathbf{u}_{h}^{n-2}, \widehat{\mathbf{E}}^{n}, \delta \widehat{\mathbf{E}}^{n+1}\right) \\
& -4 \tau \mathcal{N}\left(2 \mathbf{u}_{h}^{n}-\mathbf{u}_{h}^{n-1}, \widehat{\mathbf{E}}^{n+1}, \delta \widehat{\mathbf{E}}^{n+1}\right) .
\end{aligned}
$$

In estimating convection terms, we will use Lemma 10 frequently without notice. We recall $\|\mathbf{u}(t)\|_{2} \leq C$ to obtain

$$
\begin{aligned}
A_{1,1}+ & A_{1,2} \\
\leq & C \tau\left(\left\|\delta \delta \mathbf{u}\left(t^{n+1}\right)\right\|_{0}\left\|\mathbf{u}\left(t^{n+1}\right)\right\|_{2}\right. \\
& \left.+\left\|\delta \delta \mathbf{u}\left(t^{n}\right)\right\|_{0}\left\|\mathbf{u}\left(t^{n}\right)\right\|_{2}\right)\left\|\delta \widehat{\mathbf{E}}_{h}^{n+1}\right\|_{1} \\
\leq & \frac{\mu \tau}{6}\left\|\nabla \delta \widehat{\mathbf{E}}_{h}^{n+1}\right\|_{0}^{2} \\
& +\frac{C \tau^{4}}{\mu} \int_{t^{n-2}}^{t^{n+1}}\left\|\mathbf{u}_{t t}(t)\right\|_{0}^{2} d t .
\end{aligned}
$$

The result in Lemma 13, $\left\|2 \mathbf{E}^{n}-\mathbf{E}^{n-1}\right\|_{0} \leq C(\tau+h)$, is essential to treat the next 2 convection terms. Invoking (33), we have

$$
\begin{aligned}
A_{1,3} & +A_{1,4} \\
\leq & C \tau\left\|2 \mathbf{E}^{n}-\mathbf{E}^{n-1}\right\|_{0} \\
& \times\left\|\delta \mathbf{u}\left(t^{n+1}\right)\right\|_{2}\left\|\delta \widehat{\mathbf{E}}_{h}^{n+1}\right\|_{1} \\
& +C \tau\left\|2 \delta \mathbf{E}^{n}-\delta \mathbf{E}^{n-1}\right\|_{0} \\
& \times\left\|\mathbf{u}\left(t^{n}\right)\right\|_{2}\left\|\delta \widehat{\mathbf{E}}_{h}^{n+1}\right\|_{1} \\
\leq & \frac{\mu \tau}{6}\left\|\nabla \delta \widehat{\mathbf{E}}_{h}^{n+1}\right\|_{0}^{2} \\
& +\frac{C \tau}{\mu}\left\|2 \delta \mathbf{E}^{n}-\delta \mathbf{E}^{n-1}\right\|_{0}^{2} \\
& +\frac{C \tau^{2}\left(\tau^{2}+h^{2}\right)}{\mu} \\
& \times \int_{t_{n-1}}^{n}\left\|\mathbf{u}_{t}(t)\right\|_{2}^{2} d t .
\end{aligned}
$$

We note $\mathcal{N}\left(2 \mathbf{u}_{h}^{n}-\mathbf{u}_{h}^{n-1}, \delta \widehat{\mathbf{E}}_{h}^{n+1}, \delta \widehat{\mathbf{E}}_{h}^{n+1}\right)=0$ which comes from (30). Then we obtain

$$
\begin{aligned}
& A_{1,5}+A_{1,6} \\
& =-4 \tau \mathcal{N}\left(2 \delta \mathbf{u}_{h}^{n}-\delta \mathbf{u}_{h}^{n-1},\right. \\
& \left.\mathbf{G}^{n+1}+\widehat{\mathbf{E}}_{h}^{n+1}, \delta \widehat{\mathbf{E}}_{h}^{n+1}\right) \\
& -4 \tau \mathcal{N}\left(2 \mathbf{u}_{h}^{n-1}-\mathbf{u}_{h}^{n-2},\right. \\
& \left.\delta \mathbf{G}^{n+1}, \delta \widehat{\mathbf{E}}_{h}^{n+1}\right) \\
& =4 \tau \mathcal{N}\left(2 \delta \mathbf{E}^{n}-\delta \mathbf{E}^{n-1}-2 \delta \mathbf{u}\left(t^{n}\right)\right. \\
& \left.+\delta \mathbf{u}\left(t^{n-1}\right), \mathbf{G}^{n+1}+\widehat{\mathbf{E}}_{h}^{n+1}, \delta \widehat{\mathbf{E}}_{h}^{n+1}\right) \\
& +4 \tau \mathcal{N}\left(2 \mathbf{E}^{n-1}-\mathbf{E}^{n-2}\right. \\
& -2 \mathbf{u}\left(t^{n-1}\right)+\mathbf{u}\left(t^{n-2}\right), \\
& \left.\delta \mathbf{G}^{n+1}, \delta \widehat{\mathbf{E}}_{h}^{n+1}\right) \\
& =B_{1}+B_{2}
\end{aligned}
$$


To attack $B_{1}$, we first note Lemma 5 which is, for any $\mathbf{w}_{h} \in \mathbb{V}_{h}$, $\left\|\mathbf{w}_{h}\right\|_{\mathbf{L}^{3}(\Omega)} \leq C h^{-d / 6}\left\|\mathbf{w}_{h}\right\|_{0}$. If we apply

$$
\begin{aligned}
\| \widehat{\mathbf{E}}^{n+1} & +\mathbf{G}^{n+1} \|_{0} \\
& +\sqrt{\tau+h}\left\|\widehat{\mathbf{E}}^{n+1}+\mathbf{G}^{n+1}\right\|_{1} \\
\leq & C(\tau+h)
\end{aligned}
$$

which is the result of Lemma 13, then we can conclude, in light of (34),

$$
\begin{aligned}
B_{1} \leq & C \tau\left\|2 \delta \mathbf{E}^{n}-\delta \mathbf{E}^{n-1}\right\|_{\mathbf{L}^{3}(\Omega)} \\
& \times\left\|\widehat{\mathbf{E}}_{h}^{n+1}+\mathbf{G}^{n+1}\right\|_{1}\left\|\nabla \delta \widehat{\mathbf{E}}_{h}^{n+1}\right\|_{0} \\
& +C \tau\left\|2 \delta \mathbf{u}\left(t^{n}\right)-\delta \mathbf{u}\left(t^{n-1}\right)\right\|_{2} \\
& \times\left\|\widehat{\mathbf{E}}_{h}^{n+1}+\mathbf{G}^{n+1}\right\|_{0}\left\|\nabla \delta \widehat{\mathbf{E}}_{h}^{n+1}\right\|_{0} \\
\leq & \frac{\mu \tau}{6}\left\|\nabla \delta \widehat{\mathbf{E}}_{h}^{n+1}\right\|_{0}^{2}+\frac{C \tau}{\mu}\left(1+\frac{\tau}{h}\right) \\
& \times\left\|2 \delta \mathbf{E}^{n}-\delta \mathbf{E}^{n-1}\right\|_{0}^{2} \\
& +\frac{C \tau^{2}}{\mu}(\tau+h)^{2} \int_{t^{n-2}}^{t^{n}}\left\|\mathbf{u}_{t}(t)\right\|_{2}^{2} d t .
\end{aligned}
$$

We now estimate $B_{2}$ using $\left\|2 \mathbf{E}^{n-1}-\mathbf{E}^{n-2}\right\|_{\mathbf{L}^{3}(\Omega)} \leq$ $C h^{-d / 6}\left\|2 \mathbf{E}^{n-1}-\mathbf{E}^{n-2}\right\|_{0} \leq M$ :

$$
\begin{aligned}
B_{2} \leq & C \tau\left\|2 \mathbf{E}^{n-1}-\mathbf{E}^{n-2}\right\|_{\mathbf{L}^{3}(\Omega)} \\
& \times\left\|\delta \mathbf{G}^{n+1}\right\|_{1}\left\|\nabla \delta \widehat{\mathbf{E}}_{h}^{n+1}\right\|_{0} \\
& +C \tau\left\|2 \mathbf{u}\left(t^{n-1}\right)-\mathbf{u}\left(t^{n-2}\right)\right\|_{1} \\
& \times\left\|\delta \mathbf{G}^{n+1}\right\|_{1}\left\|\nabla \delta \widehat{\mathbf{E}}_{h}^{n+1}\right\|_{0} \\
\leq & C \tau\left\|\delta \mathbf{G}^{n+1}\right\|_{1}^{2}+\frac{\mu \tau}{6}\left\|\nabla \delta \widehat{\mathbf{E}}_{h}^{n+1}\right\|_{0}^{2} \\
\leq & \frac{\mu \tau}{6}\left\|\nabla \delta \widehat{\mathbf{E}}_{h}^{n+1}\right\|_{0}^{2}+C \tau^{2} h^{2} \\
& \times \int_{t^{n-1}}^{t^{n+1}}\left(\left\|\mathbf{u}_{t}(t)\right\|_{2}^{2}+\left\|p_{t}(t)\right\|_{1}^{2}\right) d t .
\end{aligned}
$$

In light of Hölder inequality, (51) yields

$$
\begin{aligned}
A_{2}= & -2\left\langle 3 \delta \mathbf{G}^{n+1}-4 \delta \mathbf{G}^{n}\right. \\
& \left.+\delta \mathbf{G}^{n-1}, \delta \widehat{\mathbf{E}}_{h}^{n+1}\right\rangle \\
\leq & \frac{\mu \tau}{6}\left\|\nabla \delta \mathbf{E}_{h}^{n+1}\right\|_{0}^{2} \\
& +C h^{4} \int_{t^{n-2}}^{t^{n+1}}\left(\left\|\mathbf{u}_{t}(t)\right\|_{2}^{2}\right. \\
& \left.+\left\|p_{t}(t)\right\|_{1}^{2}\right) d t .
\end{aligned}
$$

Integral by parts leads to

$$
\begin{aligned}
A_{3}= & 4 \tau\left\langle\delta \delta P_{h}^{n+1}, \nabla \cdot \delta \widehat{\mathbf{E}}_{h}^{n+1}\right\rangle \\
\leq & \frac{\mu \tau}{6}\left\|\nabla \delta \widehat{\mathbf{E}}_{h}^{n+1}\right\|_{0}^{2}+\frac{C \tau^{4}}{\mu} \\
& \times \int_{t^{n-1}}^{t^{n+1}}\left(\left\|\mathbf{u}_{t t}(t)\right\|_{2}^{2}+\left\|p_{t t}(t)\right\|_{1}^{2}\right) d t .
\end{aligned}
$$

In order to tackle $A_{4}$, marking use of $\delta \widehat{\mathbf{E}}_{h}^{n+1}=\delta \mathbf{E}_{h}^{n+1}+$ $\nabla \delta \delta \psi_{h}^{n+1}$, we readily get

$$
\begin{gathered}
A_{4}=-4 \tau\left\langle\nabla \delta \varepsilon_{h}^{n}, \delta \mathbf{E}_{h}^{n+1}+\nabla \delta \delta \psi_{h}^{n+1}\right\rangle \\
=-\frac{8 \tau^{2}}{3}\left\langle\nabla \delta \varepsilon_{h}^{n}, \nabla\left(\delta \delta \varepsilon_{h}^{n+1}-\delta \delta P_{h}^{n+1}\right)\right\rangle \\
\leq-\frac{4 \tau^{2}}{3}\left(\left\|\nabla \delta \varepsilon_{h}^{n+1}\right\|_{0}^{2}-\left\|\nabla \delta \varepsilon_{h}^{n}\right\|_{0}^{2}\right. \\
\left.-\left\|\nabla \delta \delta \varepsilon_{h}^{n+1}\right\|_{0}^{2}\right) \\
+C \tau^{3}\left\|\nabla \delta \varepsilon_{h}^{n}\right\|_{0}^{2}+C \tau\left\|\nabla \delta \delta P_{h}^{n+1}\right\|_{0}^{2} .
\end{gathered}
$$

If we now apply inequality $(a+b)^{2} \leq 4 a^{2}+(4 / 3) b^{2}$, then we can have

$$
\begin{aligned}
\frac{4 \tau^{2}}{3} \| & \nabla \delta \delta \varepsilon_{h}^{n+1} \|_{0}^{2} \\
= & \frac{4 \tau^{2}}{3}\left\|\nabla \delta \delta P_{h}^{n+1}+\frac{3}{2 \tau} \nabla \delta \delta \psi_{h}^{n+1}\right\|_{0}^{2} \\
\leq & C \tau^{2}\left\|\nabla \delta \delta P_{h}^{n+1}\right\|_{0}^{2} \\
& +4\left\|\nabla \delta \delta \psi_{h}^{n+1}\right\|_{0}^{2} .
\end{aligned}
$$

So we arrive at

$$
\begin{aligned}
A_{4} \leq & -\frac{4 \tau^{2}}{3}\left(\left\|\nabla \delta \varepsilon_{h}^{n+1}\right\|_{0}^{2}-\left\|\nabla \delta \varepsilon_{h}^{n}\right\|_{0}^{2}\right) \\
& +4\left\|\nabla \delta \delta \psi_{h}^{n+1}\right\|_{0}^{2}+C \tau^{3}\left\|\nabla \delta \varepsilon_{h}^{n}\right\|_{0}^{2} \\
& +C \tau^{4} \int_{t^{n-1}}^{t^{n+1}}\left(\left\|\mathbf{u}_{t t}(t)\right\|_{2}^{2}+\left\|p_{t t}(t)\right\|_{1}^{2}\right) d t
\end{aligned}
$$

Invoking (9), (37) and (60) lead to

$$
\begin{aligned}
A_{5}= & -4 \mu \tau\left\langle\delta q_{h}^{n}, \delta \delta q_{h}^{n+1}\right\rangle \\
= & -2 \mu \tau\left(\left\|\delta q_{h}^{n+1}\right\|_{0}^{2}-\left\|\delta q_{h}^{n}\right\|_{0}^{2}\right. \\
& \left.-\left\|\delta \delta q_{h}^{n+1}\right\|_{0}^{2}\right) \\
\leq & -2 \mu \tau\left(\left\|\delta q_{h}^{n+1}\right\|_{0}^{2}-\left\|\delta q_{h}^{n}\right\|_{0}^{2}\right) \\
& +2 \mu \tau\left\|\nabla \delta \widehat{\mathbf{E}}_{h}^{n+1}\right\|_{0}^{2} .
\end{aligned}
$$


Finally, the $A_{6}$ term becomes

$$
\begin{aligned}
A_{6} \leq & C \tau\left\|\delta \mathbf{R}^{n+1}\right\|_{0}\left\|\delta \widehat{\mathbf{E}}_{h}^{n+1}\right\|_{0} \\
\leq & C \tau\left\|\delta \widehat{\mathbf{E}}_{h}^{n+1}\right\|_{0}^{2} \\
& +C \tau^{4} \int_{t^{n-1}}^{t^{n+1}}\left\|\mathbf{u}_{t t t}(t)\right\|_{0}^{2} d t .
\end{aligned}
$$

For the new term $A_{7}$, we argue as in Lemma 13 to arrive at

$$
\begin{aligned}
A_{7} \leq & C \kappa^{2} \mu^{4} \tau \\
& \times\left(\left\|2 \delta \vartheta_{h}^{n}-\delta 9_{h}^{n-1}\right\|_{0}^{2}\right. \\
& \left.\quad+\left\|2 \delta \eta^{n}-\delta \eta^{n-1}\right\|_{0}^{2}\right) \\
& +\tau\left\|\delta \mathbf{E}_{h}^{n+1}\right\|_{0}^{2}+\frac{1}{2}\left\|\nabla \delta \delta \psi_{h}^{n+1}\right\|_{0}^{2} \\
& +C \tau^{4} \int_{t^{n-2}}^{t^{n}+1}\left\|\theta_{t t}(t)\right\|_{0}^{2} d t .
\end{aligned}
$$

We now insert the above estimates into (93) to obtain

$$
\begin{aligned}
& \delta\left\|\delta \mathbf{E}_{h}^{n+1}\right\|_{0}^{2}+\delta\left\|2 \delta \mathbf{E}_{h}^{n+1}-\delta \mathbf{E}_{h}^{n}\right\|_{0}^{2} \\
& +\left\|\delta \delta \delta \mathbf{E}_{h}^{n+1}\right\|_{0}^{2}+\mu \tau\left\|\nabla \delta \widehat{\mathbf{E}}_{h}^{n+1}\right\|_{0}^{2} \\
& +\left\|\nabla \delta \delta \psi_{h}^{n+1}\right\|_{0}^{2}+2 \mu \tau\left\|\delta q_{h}^{n+1}\right\|_{0}^{2} \\
& +\frac{4 \tau^{2}}{3}\left\|\nabla \delta \varepsilon_{h}^{n+1}\right\|_{0}^{2} \\
& \leq \frac{C \tau}{\mu}\left(\left\|2 \delta \mathbf{E}_{h}^{n}-\delta \mathbf{E}_{h}^{n-1}\right\|_{0}^{2}\right. \\
& \left.+\left\|2 \delta \mathbf{G}^{n}-\delta \mathbf{G}^{n-1}\right\|_{0}^{2}\right) \\
& +\frac{4 \tau^{2}}{3}\left\|\nabla \delta \varepsilon_{h}^{n}\right\|_{0}^{2}+C \kappa^{2} \mu^{4} \tau \\
& \times\left(\left\|2 \delta \vartheta_{h}^{n}-\delta \vartheta_{h}^{n-1}\right\|_{0}^{2}\right. \\
& \left.+\left\|2 \delta \eta^{n}-\delta \eta^{n-1}\right\|_{0}^{2}\right) \\
& +\tau\left\|\delta \mathbf{E}_{h}^{n+1}\right\|_{0}^{2}+2 \mu \tau\left\|\delta q_{h}^{n}\right\|_{0}^{2}+C \tau^{4} \\
& \times \int_{t^{n-1}}^{t^{n+1}}\left(\left\|\mathbf{u}_{t t t}(t)\right\|_{0}^{2}+\left\|\mathbf{u}_{t t}(t)\right\|_{2}^{2}\right. \\
& \left.+\left\|p_{t t}(t)\right\|_{1}^{2}+\left\|\theta_{t t}(t)\right\|_{0}^{2}\right) d t \\
& +C \tau^{3}\left\|\nabla \delta \varepsilon_{h}^{n}\right\|_{0}^{2}+C\left(\tau^{2}+h^{2}\right)^{2} \\
& \times \int_{t^{n-2}}^{t^{n+1}}\left(\left\|\mathbf{u}_{t t}(t)\right\|_{0}^{2}+\left\|\mathbf{u}_{t}(t)\right\|_{2}^{2}\right. \\
& \left.+\left\|p_{t}(t)\right\|_{1}^{2}\right) d t
\end{aligned}
$$

To evaluate errors of $\delta \vartheta_{h}^{n+1}$, we subtract two consecutive formulas (83) and choose by $\phi_{h}=4 \tau \delta \vartheta_{h}^{n+1}=4 \tau\left(\delta \vartheta^{n+1}-\right.$ $\left.\delta \eta^{n+1}\right)$ as follows:

$$
\begin{aligned}
& \delta\left\|\delta \vartheta_{h}^{n+1}\right\|_{0}^{2}+\delta\left\|2 \delta \vartheta_{h}^{n+1}\right\|_{0}^{2} \\
& \quad+\left\|\delta \delta \delta \vartheta_{h}^{n+1}\right\|_{0}^{2}+4 \lambda \mu \tau\left\|\nabla \delta \vartheta_{h}^{n+1}\right\|_{0}^{2} \\
& =A_{8}+A_{9},
\end{aligned}
$$

where

$$
\begin{aligned}
A_{8}:= & 4 \tau \mathcal{N}\left(\mathbf{u}\left(t^{n}\right), \theta\left(t^{n}\right), \delta \vartheta_{h}^{n+1}\right) \\
& -4 \tau \mathcal{N}\left(\mathbf{u}_{h}^{n}, \theta_{h}^{n}, \delta \vartheta_{h}^{n+1}\right) \\
& -4 \tau \mathcal{N}\left(\mathbf{u}\left(t^{n+1}\right), \theta\left(t^{n+1}\right), \delta \vartheta_{h}^{n+1}\right) \\
& +4 \tau \mathcal{N}\left(\mathbf{u}_{h}^{n+1}, \theta_{h}^{n+1}, \delta \vartheta_{h}^{n+1}\right) \\
A_{9}:= & -2\left\langle 3 \delta \eta^{n+1}-4 \delta \eta^{n}\right. \\
& \left.+\delta \eta^{n-1}, \delta \vartheta_{h}^{n+1}\right\rangle \\
& +4 \tau\left\langle\delta Q^{n+1}, \delta \vartheta_{h}^{n+1}\right\rangle .
\end{aligned}
$$

We treat the $A_{8}$ term first by rewriting it as follows:

$$
\begin{aligned}
A_{8}= & -4 \tau \mathcal{N}\left(\mathbf{E}^{n+1}, \theta\left(t^{n+1}\right), \delta \vartheta_{h}^{n+1}\right) \\
& -4 \tau \mathcal{N}\left(\mathbf{u}_{h}^{n+1}, \vartheta^{n+1}, \delta \vartheta_{h}^{n+1}\right) \\
& +4 \tau \mathcal{N}\left(\mathbf{E}^{n}, \theta\left(t^{n}\right), \delta \vartheta_{h}^{n+1}\right) \\
& +4 \tau \mathcal{N}\left(\mathbf{u}_{h}^{n}, \vartheta^{n}, \delta \vartheta_{h}^{n+1}\right) \\
:= & \sum_{i=1}^{4} A_{8, i} .
\end{aligned}
$$

In estimating convection terms, we will use Lemma 10 frequently without notice. We recall $\|\theta(t)\|_{2} \leq C$ and $\left\|\mathbf{E}^{n}\right\|_{0} \leq$ $C(\tau+h)$ which is the result in Lemma 13 to obtain

$$
\begin{aligned}
A_{8,1}+ & A_{8,3} \\
= & -4 \tau \mathcal{N}\left(\delta \mathbf{E}^{n+1}, \theta\left(t^{n+1}\right), \delta \vartheta_{h}^{n+1}\right) \\
& -4 \tau \mathcal{N}\left(\mathbf{E}^{n}, \delta \theta\left(t^{n+1}\right), \delta \vartheta_{h}^{n+1}\right) \\
\leq & C \tau\left(\left\|\delta \mathbf{E}^{n+1}\right\|_{0}\left\|\theta\left(t^{n+1}\right)\right\|_{2}\right. \\
& \left.+\left\|\mathbf{E}^{n}\right\|_{0}\left\|\delta \theta\left(t^{n+1}\right)\right\|_{2}\right)\left\|\delta \vartheta_{h}^{n+1}\right\|_{1} \\
\leq & \lambda \mu \tau\left\|\nabla \delta \vartheta_{h}^{n+1}\right\|_{0}^{2} \\
& +\frac{C \tau}{\lambda \mu}\left\|\delta \mathbf{E}^{n+1}\right\|_{0}^{2}+\frac{C \tau^{2}(\tau+h)^{2}}{\lambda \mu} \\
& \times \int_{t^{n}}^{t^{n+1}}\left\|\theta_{t}(t)\right\|_{2}^{2} d t .
\end{aligned}
$$


In conjunction with $\mathcal{N}\left(\mathbf{u}_{h}^{n+1}, \delta \vartheta_{h}^{n+1}, \delta \vartheta_{h}^{n+1}\right)=0$ which comes from (30), we rewrite $A_{8,2}+A_{8,4}$ as

$$
\begin{aligned}
A_{8,2}+ & A_{8,4} \\
= & 4 \tau \mathcal{N}\left(\mathbf{E}_{h}^{n+1}-\mathbf{U}_{h}^{n+1},\right. \\
& \left.\delta \eta^{n+1}, \delta \vartheta_{h}^{n+1}\right) \\
& +4 \tau \mathcal{N}\left(\delta \mathbf{E}_{h}^{n+1}-\delta \mathbf{U}_{h}^{n+1},\right. \\
\left.\vartheta^{n}, \delta \vartheta_{h}^{n+1}\right) & \\
:= & B_{3}+B_{4} .
\end{aligned}
$$

Because we can readily get $\left\|\mathbf{E}_{h}^{n+1}-\mathbf{U}_{h}^{n+1}\right\|_{\mathbf{L}^{3}(\Omega)} \leq M$ via Lemmas 5 and 13, we conclude, in conjunction with (60),

$$
\begin{aligned}
B_{3} \leq & C \tau\left\|\mathbf{E}_{h}^{n+1}-\mathbf{U}_{h}^{n+1}\right\|_{\mathbf{L}^{3}(\Omega)} \\
& \times\left\|\delta \eta^{n+1}\right\|_{1}\left\|\delta \vartheta_{h}^{n+1}\right\|_{1} \\
\leq & \lambda \mu \tau\left\|\nabla \delta 9_{h}^{n+1}\right\|_{0}^{2}+\frac{C \tau^{2} h^{2}}{\lambda \mu} \\
& \times \int_{t^{n}}^{t^{n+1}}\left\|\theta_{t}(t)\right\|_{2}^{2} d t .
\end{aligned}
$$

Before we attack $B_{4}$, we note that the assumption $\tau \leq C h$ is required to apply $\left\|\delta \mathrm{E}_{h}^{n}\right\|_{\mathbf{L}^{3}(\Omega)} \leq C \tau^{-1 / 2}\left\|\delta \mathbf{E}_{h}^{n}\right\|_{0}$. We now imply $\left\|\vartheta^{n}\right\|_{0}^{2}+\tau\left\|\vartheta^{n}\right\|_{1}^{2} \leq C\left(\tau^{2}+h^{2}\right)$ and (51) to get

$$
\begin{aligned}
B_{4}= & 4 \tau \mathcal{N}\left(\delta \mathbf{G}^{n+1}-\delta \mathbf{u}\left(t^{n+1}\right)\right. \\
& \left.+\delta \mathbf{E}_{h}^{n+1}, \vartheta^{n}, \delta \vartheta_{h}^{n+1}\right) \\
\leq & C \tau\left(\left(\left\|\delta \mathbf{G}^{n+1}\right\|_{1}+\left\|\delta \mathbf{E}_{h}^{n+1}\right\|_{\mathbf{L}^{3}(\Omega)}\right)\right. \\
& \left.\times\left\|\vartheta^{n}\right\|_{1}+\left\|\delta \mathbf{u}\left(t^{n}\right)\right\|_{2}\left\|\vartheta^{n}\right\|_{0}\right)\left\|\delta \vartheta_{h}^{n+1}\right\|_{1} \\
\leq & \frac{C \tau}{\lambda \mu}\left\|\delta \mathbf{E}_{h}^{n+1}\right\|_{0}^{2}+\lambda \mu \tau\left\|\nabla \delta \vartheta_{h}^{n+1}\right\|_{0}^{2} \\
& +\frac{C \tau^{2}\left(\tau^{2}+h^{2}\right)}{\lambda \mu} \\
& \times \int_{t^{n-1}}^{t^{n+1}}\left(\left\|\mathbf{u}_{t}(t)\right\|_{2}^{2}+\left\|p_{t}(t)\right\|_{1}^{2}\right) d t .
\end{aligned}
$$

In light of Hölder inequality, $A_{9}$ becomes

$$
\begin{aligned}
A_{9} \leq & \mathrm{C} \tau \\
& \left\|\delta \vartheta_{h}^{n+1}\right\|_{0}^{2}+C\left(\tau^{4}+h^{4}\right) \\
& \times \int_{t^{n-2}}^{t^{n+1}}\left(\left\|\theta_{t}(t)\right\|_{2}^{2}+\left\|\theta_{t t t}\right\|_{0}^{2}\right) d t .
\end{aligned}
$$

Inserting the above estimates into (111) yields

$$
\begin{gathered}
\delta\left\|\delta \vartheta_{h}^{n+1}\right\|_{0}^{2}+\delta\left\|2 \delta 9_{h}^{n+1}-\delta 9_{h}^{n}\right\|_{0}^{2} \\
+\left\|\delta \delta \delta \vartheta_{h}^{n+1}\right\|_{0}^{2}+\lambda \mu \tau\left\|\nabla \delta \vartheta_{h}^{n+1}\right\|_{0}^{2} \\
\leq C \tau\left\|\delta \vartheta_{h}^{n+1}\right\|_{0}^{2} \\
+\frac{C \tau}{\lambda \mu}\left(\left\|\delta \mathbf{E}_{h}^{n+1}\right\|_{0}+\left\|\delta \mathbf{E}_{h}^{n}\right\|_{0}^{2}\right. \\
\left.+\left\|\delta \mathbf{G}^{n+1}\right\|_{0}\right)+C\left(\tau^{2}+h^{2}\right)^{2} \\
\times \int_{t^{n-2}}^{t^{n+1}}\left(\left\|\mathbf{u}_{t}(t)\right\|_{2}^{2}+\left\|p_{t}(t)\right\|_{1}^{2}\right. \\
\left.+\left\|\theta_{t}(t)\right\|_{2}^{2}+\left\|\theta_{t t t}\right\|_{0}^{2}\right) d t .
\end{gathered}
$$

Adding 2 equations (110) and (119) and then summing up $n$ from 2 to $N$ lead up to (92) and complete the proof.

We now estimate optimal accuracy for velocity and temperature.

Lemma 17 (full rate of convergence for velocity and temperature). One denotes that $\left(\mathbf{v}^{n+1}, r^{n+1}\right)$ and $\left(\mathbf{v}_{h}^{n+1}, r_{h}^{n+1}\right)$ are the solutions of (16) and (24) with $\mathbf{z}=\mathbf{E}_{h}^{n+1}$, respectively. And let $\omega^{n+1}$ and $\omega_{h}^{n+1}$ be solutions of (17) and (28) with $\xi=\vartheta_{h}^{n+1}$. Let the exact solution of (1) be smooth enough and $\tau \leq$ Ch. If Assumptions 1 and 3-5 hold, then one has

$$
\begin{aligned}
& \left\|\nabla \mathbf{v}_{h}^{N+1}\right\|_{0}^{2} \\
& +\left\|\nabla\left(2 \mathbf{v}_{h}^{N+1}-\mathbf{v}_{h}^{N}\right)\right\|_{0}^{2}+\left\|\nabla \omega_{h}^{N+1}\right\|_{0}^{2} \\
& +\left\|\nabla\left(2 \omega_{h}^{N+1}-\omega_{h}^{N}\right)\right\|_{0}^{2} \\
& +\sum_{n=1}^{N}\left(\left\|\nabla \delta \delta \mathbf{v}_{h}^{n+1}\right\|_{0}^{2}\right. \\
& \left.+\left\|\nabla \delta \delta \omega_{h}^{n+1}\right\|_{0}^{2}\right) \\
& +2 \mu \tau \sum_{n=1}^{N}\left(\left\|\mathbf{E}_{h}^{n+1}\right\|_{0}^{2}\right. \\
& \left.+\lambda\left\|\vartheta_{h}^{n+1}\right\|_{0}^{2}\right) \\
& \leq C\left(\tau^{4}+h^{4}\right) .
\end{aligned}
$$

Proof. We choose $\mathbf{w}_{h}=4 \tau \mathbf{v}_{h}^{n+1} \in \mathbb{V}_{h}$ in (66) to obtain

$$
\begin{aligned}
\delta \| & \nabla \mathbf{v}_{h}^{n+1}\left\|_{0}^{2}+\delta\right\| \nabla\left(2 \mathbf{v}_{h}^{n+1}-\mathbf{v}_{h}^{n}\right) \|_{0}^{2} \\
& +\left\|\nabla \delta \delta \mathbf{v}_{h}^{n+1}\right\|_{0}^{2}+4 \mu \tau\left\|\mathbf{E}_{h}^{n+1}\right\|_{0}^{2} \\
= & \sum_{i=1}^{5} A_{i},
\end{aligned}
$$


where

$$
\begin{gathered}
A_{1}:=4 \tau \mathcal{N}\left(2 \mathbf{u}_{h}^{n}-\mathbf{u}_{h}^{n-1}, \widehat{\mathbf{u}}_{h}^{n+1}, \mathbf{v}_{h}^{n+1}\right) \\
-4 \tau \mathcal{N}\left(\mathbf{u}\left(t^{n+1}\right), \mathbf{u}\left(t^{n+1}\right), \mathbf{v}_{h}^{n+1}\right), \\
A_{2}:=\left\langle 3 \mathbf{G}^{n+1}-4 \mathbf{G}^{n}+\mathbf{G}^{n-1}, \mathbf{v}_{h}^{n+1}\right\rangle, \\
A_{3}:=4 \mu \tau\left\langle\widehat{\mathbf{E}}_{h}^{n+1}, \nabla r_{h}^{n+1}\right\rangle, \\
A_{4}:=4 \tau\left\langle\mathbf{R}^{n+1}, \mathbf{v}_{h}^{n+1}\right\rangle, \\
A_{5}:=4 \kappa \mu^{2} \tau\left\langle\mathbf{g}\left(\theta\left(t^{n+1}\right)-2 \theta_{h}^{n}+\theta_{h}^{n-1}\right), \mathbf{v}_{h}^{n+1}\right\rangle .
\end{gathered}
$$

We now estimate all the terms from $A_{1}$ to $A_{5}$, respectively. The convection term $A_{1}$ can be rewritten as follows:

$$
\begin{aligned}
A_{1}= & -4 \tau \mathcal{N}\left(\delta \delta \mathbf{u}\left(t^{n+1}\right), \mathbf{u}\left(t^{n+1}\right), \mathbf{v}_{h}^{n+1}\right) \\
& -4 \tau \mathcal{N}\left(2 \mathbf{u}\left(t^{n}\right)-\mathbf{u}\left(t^{n-1}\right), \widehat{\mathbf{E}}^{n+1}, \mathbf{v}_{h}^{n+1}\right) \\
& +4 \tau \mathcal{N}\left(2 \mathbf{E}^{n}-\mathbf{E}^{n-1}, \widehat{\mathbf{E}}^{n+1}, \mathbf{v}_{h}^{n+1}\right) \\
& -4 \tau \mathcal{N}\left(2 \mathbf{E}^{n}-\mathbf{E}^{n-1}, \mathbf{u}\left(t^{n+1}\right), \mathbf{v}_{h}^{n+1}\right)
\end{aligned}
$$

and we denote by $A_{1, i}$, for $i=1,2, \ldots, 4$, the four terms in the right-hand side. To estimate convection terms, we will use frequently Lemma 10 without notice. Using $\left\|\mathbf{u}\left(t^{n+1}\right)\right\|_{2} \leq M$, we can readily get

$$
\begin{aligned}
A_{1,1} \leq & C \tau\left\|\delta \delta \mathbf{u}\left(t^{n+1}\right)\right\|_{0} \\
& \times\left\|\mathbf{u}\left(t^{n+1}\right)\right\|_{2}\left\|\mathbf{v}_{h}^{n+1}\right\|_{1} \\
\leq & C \tau\left\|\nabla \mathbf{v}_{h}^{n+1}\right\|_{0}^{2}+C \mu \tau^{4} \\
& \times \int_{t^{n-1}}^{t^{n+1}}\left\|\mathbf{u}_{t t}(t)\right\|_{0}^{2} d t, \\
A_{1,4} \leq & C \tau\left(\left\|\mathbf{E}^{n}\right\|_{0}+\left\|\delta \mathbf{E}^{n}\right\|_{0}\right) \\
& \times\left\|\mathbf{u}\left(t^{n+1}\right)\right\|\left\|_{2}\right\| \mathbf{v}_{h}^{n+1} \|_{1} \\
\leq & \frac{\mu \tau}{2}\left(\left\|\mathbf{E}_{h}^{n}\right\|_{0}^{2}+\left\|\mathbf{G}^{n}\right\|_{0}^{2}\right. \\
& \left.+\left\|\delta \mathbf{E}_{h}^{n}\right\|_{0}^{2}+\left\|\delta \mathbf{G}^{n}\right\|_{0}^{2}\right) \\
+ & \frac{C \tau}{\mu}\left\|\nabla \mathbf{v}_{h}^{n+1}\right\|_{0}^{2} .
\end{aligned}
$$

Because $\nabla \cdot\left(2 \mathbf{u}\left(t^{n}\right)-\mathbf{u}\left(t^{n-1}\right)\right)=0$ and $2 \mathbf{u}\left(t^{n}\right)-\mathbf{u}\left(t^{n-1}\right)=\mathbf{0}$ on boundary, we can use (32) and so we get

$$
\begin{gathered}
A_{1,2} \leq C \tau\left\|2 \mathbf{u}\left(t^{n}\right)-\mathbf{u}\left(t^{n-1}\right)\right\|_{2} \\
\times\left\|\widehat{\mathbf{E}}^{n+1}\right\|_{0}\left\|\mathbf{v}_{h}^{n+1}\right\|_{1} \\
\leq \frac{\mu \tau}{2}\left(\left\|\mathbf{E}_{h}^{n+1}\right\|_{0}^{2}+\left\|\mathbf{G}^{n+1}\right\|_{0}^{2}\right. \\
\left.+\left\|\nabla \delta \psi_{h}^{n+1}\right\|_{0}^{2}\right) \\
+\frac{C \tau}{\mu}\left\|\nabla \mathbf{v}_{h}^{n+1}\right\|_{0}^{2} .
\end{gathered}
$$

In light of $\varepsilon_{h}^{n+1}=P_{h}^{n+1}+3 \psi_{h}^{n+1} / 2 \tau$, we can obtain

$$
\begin{aligned}
\left\|\nabla \delta \psi_{h}^{n+1}\right\|_{0}^{2} \\
=\frac{4 \tau^{2}}{9}\left\|\nabla\left(\delta \varepsilon_{h}^{n+1}-\delta P_{h}^{n+1}\right)\right\|_{0}^{2} \\
\leq C \tau^{2}\left\|\nabla \delta \varepsilon_{h}^{n+1}\right\|_{0}^{2} \\
\quad+C \tau^{3} \int_{t^{n}}^{t^{n+1}}\left(\left\|\mathbf{u}_{t}(t)\right\|_{2}^{2}+\left\|p_{t}(t)\right\|_{1}^{2}\right) d t,
\end{aligned}
$$

and so we can conclude

$$
\begin{aligned}
A_{1,2} \leq & \frac{\mu \tau}{2}\left(\left\|\mathbf{E}_{h}^{n+1}\right\|_{0}^{2}+\left\|\mathbf{G}^{n+1}\right\|_{0}^{2}\right) \\
& +C \mu \tau^{3}\left\|\nabla \delta \varepsilon_{h}^{n+1}\right\|_{0}^{2}+C \mu \tau^{4} \\
& \times \int_{t^{n}}^{t^{n+1}}\left(\left\|\mathbf{u}_{t}(t)\right\|_{2}^{2}+\left\|p_{t}(t)\right\|_{1}^{2}\right) d t \\
& +\frac{C \tau}{\mu}\left\|\nabla \mathbf{v}_{h}^{n+1}\right\|_{0}^{2} .
\end{aligned}
$$

If we apply $\left\|2 \mathbf{E}^{n}-\mathbf{E}^{n-1}\right\|_{\mathbf{L}^{3}(\Omega)} \leq C h^{-d / 6}\left\|2 \mathbf{E}^{n}-\mathbf{E}^{n-1}\right\|_{0} \leq$ $\mathrm{Ch}^{-d / 6}(\tau+h)$ which can be derived from Lemmas 5 and 13 , then we can get, by the help of Lemma 10 and Assumption 1,

$$
\begin{aligned}
A_{1,3} \leq & C \tau\left\|2 \mathbf{E}^{n}-\mathbf{E}^{n-1}\right\|_{0} \\
& \times\left\|\widehat{\mathbf{E}}^{n+1}\right\|_{1}\left\|\mathbf{v}^{n+1}\right\|_{2} \\
& +C \tau\left\|2 \mathbf{E}^{n}-\mathbf{E}^{n-1}\right\|_{\mathbf{L}^{3}(\Omega)} \\
& \times\left\|\widehat{\mathbf{E}}^{n+1}\right\|_{1}\left\|\mathbf{v}^{n+1}-\mathbf{v}_{h}^{n+1}\right\|_{1} \\
\leq & C \tau(\tau+h)\left\|\widehat{\mathbf{E}}^{n+1}\right\|_{1}\left\|\mathbf{v}^{n+1}\right\|_{2} \\
& +C \tau h\left\|\widehat{\mathbf{E}}^{n+1}\right\|_{1}\left\|\mathbf{v}^{n+1}\right\|_{2} \\
\leq & \frac{C}{\mu} \tau(\tau+h)^{2} \\
& \times\left(\left\|\nabla \widehat{\mathbf{E}}_{h}^{n+1}\right\|_{0}^{2}+\left\|\nabla \mathbf{G}^{n+1}\right\|_{0}^{2}\right) \\
& +\frac{\mu \tau}{2}\left\|\mathbf{E}_{h}^{n+1}\right\|_{0}^{2} .
\end{aligned}
$$

In conjunction with (51), we can have

$$
\begin{aligned}
A_{2} \leq & \frac{C}{\tau}\left(\left\|\delta \mathbf{G}^{n+1}\right\|_{0}^{2}+\left\|\delta \mathbf{G}^{n}\right\|_{0}^{2}\right)+C \tau\left\|\mathbf{v}_{h}^{n+1}\right\|_{0}^{2} \\
\leq & C \tau\left\|\mathbf{v}_{h}^{n+1}\right\|_{0}^{2}+C h^{4} \\
& \times \int_{t^{n-1}}^{t^{n+1}}\left(\left\|\mathbf{u}_{t}(t)\right\|_{2}^{2}+\left\|p_{t}(t)\right\|_{1}^{2}\right) d t .
\end{aligned}
$$


The definition $\widehat{\mathbf{E}}_{h}^{n+1}=\mathbf{E}_{h}^{n+1}+\nabla \delta \psi_{h}^{n+1}$ and Assumption 1 give us

$$
\begin{aligned}
A_{3} & =4 \mu \tau\left\langle\nabla \delta \psi_{h}^{n+1}, \nabla r_{h}^{n+1}\right\rangle \\
& \leq \mu \tau\left\|\mathbf{E}_{h}^{n+1}\right\|_{0}^{2}+\frac{C \tau}{\mu}\left\|\nabla \delta \psi_{h}^{n+1}\right\|_{0}^{2} .
\end{aligned}
$$

If we apply (127) again, then we arrive at

$$
\begin{aligned}
A_{3} \leq & \mu \tau\left\|\mathbf{E}_{h}^{n+1}\right\|_{0}^{2} \\
& +\frac{C \tau^{3}}{\mu}\left\|\nabla \delta \varepsilon_{h}^{n+1}\right\|_{0}^{2} \\
& +\frac{C \tau^{4}}{\mu} \int_{t^{n}}^{t^{n+1}}\left\|\nabla p_{t}(t)\right\|_{0}^{2} d t .
\end{aligned}
$$

On the other hand, the truncation error term becomes

$$
\begin{aligned}
A_{4}= & 4 \tau\left\langle\mathbf{R}^{n+1}, \mathbf{v}_{h}^{n+1}\right\rangle \\
\leq & C \tau\left\|\nabla \mathbf{v}_{h}^{n+1}\right\|_{0}^{2}+C \tau^{4} \\
& \times \int_{t^{n-1}}^{t^{n+1}}\left\|\mathbf{u}_{t t t}(s)\right\|_{0}^{2} d t .
\end{aligned}
$$

For the new term $A_{5}$, we observe $\left\|\delta \delta \theta\left(t^{n+1}\right)\right\|_{0} \leq$ $C \tau^{3} \int_{t^{n-1}}^{t^{n+1}}\left\|\theta_{t}(t)\right\|_{0}^{2} d t$; whence

$$
\begin{aligned}
A_{5}= & 4 \kappa \mu^{2} \tau \\
& \times\left\langle\mathbf{g}\left(\delta \delta \theta\left(t^{n+1}\right)+2 \vartheta^{n}-\vartheta^{n-1}\right), \mathbf{v}_{h}^{n+1}\right\rangle \\
\leq & C \tau\left\|\nabla \mathbf{v}_{h}^{n+1}\right\|_{0}^{2}+C \kappa^{2} \mu^{4} \tau \\
& \times\left\|2 \vartheta^{n}-\vartheta^{n-1}\right\|_{0}^{2}+C \kappa^{2} \mu^{4} \tau^{4} \\
& \times \int_{t^{n-1}}^{t^{n+1}}\left\|\theta_{t}(t)\right\|_{0}^{2} d t .
\end{aligned}
$$

Invoking $\mathbf{v}^{0}=\mathbf{0}$ and inserting the above estimates from $A_{1}$ and $A_{5}$ into (121) lead us to

$$
\begin{aligned}
\delta\left\|\nabla \mathbf{v}_{h}^{n+1}\right\|_{0}^{2} & \\
& +\delta\left\|\nabla\left(2 \mathbf{v}_{h}^{n+1}-\mathbf{v}_{h}^{n}\right)\right\|_{0}^{2} \\
& +\left\|\nabla \delta \delta \mathbf{v}_{h}^{n+1}\right\|_{0}^{2} \\
& +2 \mu \tau\left\|\mathbf{E}_{h}^{n+1}\right\|_{0}^{2} \\
\leq & \frac{\mu \tau}{2}\left(\left\|\mathbf{E}_{h}^{n}\right\|_{0}^{2}+\left\|\mathbf{G}^{n+1}\right\|_{0}^{2}\right. \\
& \left.\quad+\left\|\mathbf{G}^{n}\right\|_{0}^{2}+\left\|\delta \mathbf{E}_{h}^{n}\right\|_{0}^{2}+\left\|\delta \mathbf{G}^{n}\right\|_{0}^{2}\right)
\end{aligned}
$$

$$
\begin{aligned}
& +C \kappa^{2} \mu^{4} \tau\left\|2 \vartheta^{n}-\vartheta^{n-1}\right\|_{0}^{2} \\
& +\frac{C}{\mu} \tau(\tau+h)^{2} \\
& \times\left(\left\|\nabla \widehat{\mathbf{E}}_{h}^{n+1}\right\|_{0}^{2}+\left\|\nabla \mathbf{G}^{n+1}\right\|_{0}^{2}\right) \\
& +\frac{C \tau^{3}}{\mu}\left\|\nabla \delta \varepsilon_{h}^{n+1}\right\|_{0}^{2} \\
& +C \mu\left(\tau^{4}+h^{4}\right) \\
& \times \int_{t^{n-1}}^{t^{n+1}}\left(\left\|\mathbf{u}_{t}(t)\right\|_{2}^{2}+\left\|p_{t}(t)\right\|_{1}^{2}\right) d t \\
& +C \tau\left\|\nabla \mathbf{v}_{h}^{n+1}\right\|_{0}^{2}+C \tau^{4} \\
& \times \int_{t^{n-1}}^{t^{n+1}}\left(\left\|\mathbf{u}_{t t}(t)\right\|_{0}^{2}+\left\|\mathbf{u}_{t t t}(t)\right\|_{0}^{2}+\left\|\theta_{t}(t)\right\|_{0}^{2}\right) d t .
\end{aligned}
$$

On the other hand, we choose $\phi_{h}=4 \tau \omega_{h}^{n+1}$ in (83) to obtain

$$
\begin{aligned}
& \delta\left\|\nabla \omega_{h}^{n+1}\right\|_{0}^{2} \\
& +\delta\left\|\nabla\left(2 \omega_{h}^{n+1}-\omega_{h}^{n}\right)\right\|_{0}^{2} \\
& +\left\|\nabla \delta \delta \omega_{h}^{n+1}\right\|_{0}^{2} \\
& +4 \lambda \mu \tau\left\|\vartheta_{h}^{n+1}\right\|_{0}^{2} \\
& =A_{6}+A_{7},
\end{aligned}
$$

where

$$
\begin{aligned}
A_{6}:= & 4 \tau \mathcal{N}\left(\mathbf{u}_{h}^{n+1}, \theta_{h}^{n+1}, \omega_{h}^{n+1}\right) \\
& -4 \tau \mathcal{N}\left(\mathbf{u}\left(t^{n+1}\right), \theta\left(t^{n+1}\right), \omega_{h}^{n+1}\right), \\
A_{7}:= & -2\left\langle 3 \eta^{n+1}-4 \eta^{n}+\eta^{n-1}, \omega_{h}^{n+1}\right\rangle \\
& +4 \tau\left\langle Q^{n+1}, \omega_{h}^{n+1}\right\rangle .
\end{aligned}
$$

In order to estimate $A_{6}$, we note first $\left\|\mathbf{E}^{n+1}\right\|_{\mathbf{L}^{3}(\Omega)} \leq$ $C h^{-1 / 2}\left\|\mathbf{E}^{n+1}\right\|_{0}$ and $\left\|\vartheta^{n+1}\right\|_{1}^{2} \leq C\left(\left(\tau^{2}+h^{2}\right) / \tau\right)$ which come from Lemmas 5 and 13, respectively. Then Lemma 10 and the assumption $\tau \leq C h$ yield

$$
\begin{aligned}
A_{6}= & -4 \tau \mathcal{N}\left(\mathbf{E}^{n+1}, \theta\left(t^{n+1}\right), \omega_{h}^{n+1}\right) \\
& +4 \tau \mathcal{N}\left(\mathbf{E}^{n+1}-\mathbf{u}\left(t^{n+1}\right), \vartheta^{n+1}, \omega_{h}^{n+1}\right) \\
\leq & C \tau\left\|\mathbf{E}^{n+1}\right\|_{0}\left\|\theta\left(t^{n+1}\right)\right\|_{2}\left\|\omega_{h}^{n+1}\right\|_{1} \\
& +C \tau\left\|\mathbf{E}^{n+1}\right\|_{\mathbf{L}^{3}(\Omega)}\left\|\vartheta^{n+1}\right\|_{1}\left\|\omega_{h}^{n+1}\right\|_{1} \\
& +C \tau\left\|\mathbf{u}\left(t^{n+1}\right)\right\|\left\|_{2}\right\| \vartheta^{n+1}\left\|_{0}\right\|_{h}^{n+1} \|_{1} \\
\leq & C \tau\left\|\nabla \omega_{h}^{n+1}\right\|_{0}^{2}+\mu \tau\left\|\mathbf{E}^{n+1}\right\|_{0}^{2} \\
& +\lambda \mu \tau\left(\left\|\vartheta_{h}^{n+1}\right\|_{0}^{2}+\left\|\eta^{n+1}\right\|_{0}^{2}\right) .
\end{aligned}
$$


Since $\left\|\delta \eta^{n+1}\right\|_{0}^{2} \leq C h^{4} \tau \int_{n}^{n+1}\left\|\theta_{t}(t)\right\|_{2}^{2} d t$, we can readily get

$$
\begin{aligned}
A_{7} \leq & C \tau\left\|\nabla \omega_{h}^{n+1}\right\|_{0}^{2}+C\left(\tau^{4}+h^{4}\right) \\
& \times \int_{n-1}^{n+1}\left(\left\|\theta_{t}(t)\right\|_{2}^{2}+\left\|\theta_{t t t}(t)\right\|_{2}^{2}\right) d t .
\end{aligned}
$$

Inserting the above estimates into (136) yields

$$
\begin{aligned}
\delta\left\|\nabla \omega_{h}^{n+1}\right\|_{0}^{2} & \\
+ & \delta\left\|\nabla\left(2 \omega_{h}^{n+1}-\omega_{h}^{n}\right)\right\|_{0}^{2} \\
& +\left\|\nabla \delta \delta \omega_{h}^{n+1}\right\|_{0}^{2}+3 \lambda \mu \tau\left\|\vartheta_{h}^{n+1}\right\|_{0}^{2} \\
\leq & C \tau\left\|\nabla \omega_{h}^{n+1}\right\|_{0}^{2}+\lambda \mu \tau\left\|\eta^{n+1}\right\|_{0}^{2} \\
& +\mu \tau\left\|\mathbf{E}^{n+1}\right\|_{0}^{2}+C\left(\tau^{4}+h^{4}\right) \\
& \times \int_{n-1}^{n+1}\left(\left\|\theta_{t}(t)\right\|_{2}^{2}+\left\|\theta_{t t t}(t)\right\|_{2}^{2}\right) d t .
\end{aligned}
$$

In conjunction with the discrete Gronwall inequality, adding (135) and (140) and summing over $n$ from 1 to $N$ lead to $(120)$.

We now estimate the pressure error in $L^{2}\left(0, T ; L^{2}(\Omega)\right)$. This hinges on the error estimates for the time derivative of velocity and temperature of Lemma 15.

Lemma 18 (pressure error estimate). Let the exact solution of (1) be smooth enough and $\tau \leq C h$. If Assumptions 1-5 hold, then one has

$$
\tau \sum_{n=1}^{N}\left\|e_{h}^{n+1}\right\|_{0}^{2} \leq C\left(\tau^{2}+h^{2}\right) .
$$

Proof. We first recall again inf-sup condition in Assumption 2. Consequently, it suffices to estimate $\left\langle e^{n+1}, \nabla \cdot \mathbf{w}\right\rangle$ in terms of $\|\nabla \mathbf{w}\|_{0}$. In conjunction with (10), we can rewrite (66) as

$$
\begin{aligned}
\left\langle e_{h}^{n+1}, \nabla \cdot \mathbf{w}_{h}\right\rangle & \\
= & \frac{1}{2 \tau}\left\langle 3 \mathbf{E}^{n+1}-4 \mathbf{E}^{n}+\mathbf{E}^{n-1}, \mathbf{w}_{h}\right\rangle \\
& +\mu\left\langle\nabla \widehat{\mathbf{E}}_{h}^{n+1}, \nabla \mathbf{w}_{h}\right\rangle \\
& +\mathcal{N}\left(\delta \delta \mathbf{u}\left(t^{n+1}\right), \mathbf{u}\left(t^{n+1}\right), \mathbf{w}_{h}\right) \\
& +\mathcal{N}\left(2 \mathbf{u}\left(t^{n+1}\right)-\mathbf{u}\left(t^{n}\right), \widehat{\mathbf{E}}^{n+1}, \mathbf{w}_{h}\right) \\
& +\mathcal{N}\left(2 \mathbf{E}^{n}-\mathbf{E}^{n-1}, \widehat{\mathbf{u}}_{h}^{n+1}, \mathbf{w}_{h}\right) \\
& -\mu\left\langle\nabla \delta q_{h}^{n+1}, \mathbf{w}_{h}\right\rangle-\left\langle\mathbf{R}^{n+1}, \mathbf{w}_{h}\right\rangle \\
& +\kappa \mu^{2}\left\langle\mathbf{g}\left(\theta\left(t^{n+1}\right)-2 \theta_{h}^{n}+\theta_{h}^{n-1}\right), \mathbf{w}_{h}\right\rangle \\
:= & \sum_{i=1}^{8} A_{i} .
\end{aligned}
$$

We now proceed to estimate each term from $A_{1}$ to $A_{7}$ separately. We readily obtain

$$
\begin{aligned}
& A_{1} \leq \frac{C}{\tau}\left(\left\|\delta \mathbf{E}^{n+1}\right\|_{0}+\left\|\delta \mathbf{E}^{n}\right\|_{0}\right)\left\|\mathbf{w}_{h}\right\|_{0} \\
& \leq \frac{C}{\tau}\left(\left\|\delta \mathbf{E}^{n+1}\right\|_{0}+\left\|\delta \mathbf{E}^{n}\right\|_{0}\right)\left\|\nabla \mathbf{w}_{h}\right\|_{0}, \\
& A_{2} \leq C\left\|\nabla \widehat{\mathbf{E}}_{h}^{n+1}\right\|_{0}\left\|\nabla \mathbf{w}_{h}\right\|_{0} .
\end{aligned}
$$

Terms $A_{3}$ and $A_{4}$ can be dealt with thanks to the aid of Lemma 10 and $\left\|\mathbf{u}\left(t^{n+1}\right)\right\|_{2} \leq M$ as follows:

$$
\begin{aligned}
A_{3} \leq & C\left\|\delta \delta \mathbf{u}\left(t^{n+1}\right)\right\|_{0} \\
& \times\left\|\mathbf{u}\left(t^{n+1}\right)\right\|\left\|_{2}\right\| \mathbf{w}_{h} \|_{1} \\
\leq & C\left\|\delta \delta \mathbf{u}\left(t^{n+1}\right)\right\|_{1}\left\|\nabla \mathbf{w}_{h}\right\|_{1}, \\
A_{4} \leq & C\left\|2 \mathbf{u}\left(t^{n+1}\right)-\mathbf{u}\left(t^{n}\right)\right\|_{2} \\
& \times\left\|\widehat{\mathbf{E}}^{n+1}\right\|_{0}\left\|\mathbf{w}_{h}\right\|_{1} \\
\leq & C\left\|\widehat{\mathbf{E}}^{n+1}\right\|_{0}\left\|\nabla \mathbf{w}_{h}\right\|_{0} .
\end{aligned}
$$

In light of $\left\|\widehat{\mathbf{u}}_{h}^{n+1}\right\|_{1}=\left\|\widehat{\mathbf{E}}^{n+1}-\mathbf{u}\left(t^{n+1}\right)\right\|_{1} \leq C$ from Lemma 13, we can have

$$
\begin{aligned}
A_{5} \leq & C\left\|2 \mathbf{E}^{n}-\mathbf{E}^{n-1}\right\|_{\mathbf{L}^{3}(\Omega)} \\
& \times\left\|\widehat{\mathbf{u}}_{h}^{n+1}\right\|_{1}\left\|\mathbf{w}_{h}\right\|_{1} \\
\leq & \frac{C}{\sqrt{h}}\left(\left\|\mathbf{E}^{n+1}\right\|_{0}+\left\|\mathbf{E}^{n}\right\|_{0}\right) \\
& \times\left\|\nabla \mathbf{w}_{h}\right\|_{0} .
\end{aligned}
$$

Integrate by parts and Hölder inequality yield

$$
A_{6} \leq C\left\|\delta q_{h}^{n+1}\right\|_{0}\left\|\nabla \mathbf{w}_{h}\right\|_{0} .
$$

On the other hand, we have

$$
A_{7} \leq\left\|\mathbf{R}^{n+1}\right\|_{-1}\|\nabla \mathbf{w}\|_{0} .
$$

The new term $A_{8}$ can be bound by the Hölder inequality as

$$
\begin{aligned}
& A_{8}=\kappa \mu^{2} \\
& \times\langle\mathbf{g}( \delta \delta \theta\left(t^{n+1}\right) \\
&\left.\left.-2 \vartheta^{n}+\vartheta^{n-1}\right), \mathbf{w}_{h}^{n+1}\right\rangle \\
& \leq C \kappa \mu^{2}\left(\left\|\delta \delta \theta\left(t^{n+1}\right)\right\|_{0}\right. \\
&+\left\|2 \vartheta_{h}^{n}-\vartheta_{h}^{n-1}\right\|_{0} \\
&\left.+\left\|2 \eta^{n}-\eta^{n-1}\right\|_{0}\right)\|\nabla \mathbf{w}\|_{0} .
\end{aligned}
$$


TABLE 1: Error decay for Algorithm 1 with $\tau=h$ and $\mu=1$.

\begin{tabular}{|c|c|c|c|c|c|}
\hline$\tau=h$ & $1 / 16$ & $1 / 32$ & $1 / 64$ & $1 / 128$ & $1 / 256$ \\
\hline \multirow{2}{*}{$\|E\|_{0}$} & 0.00198001 & 0.000651734 & 0.000188113 & $5.0822 e-05$ & $1.32686 e-05$ \\
\hline & Order & 1.603153 & 1.792684 & 1.888075 & 1.937437 \\
\hline \multirow{2}{*}{$\|E\|_{L^{\infty}}$} & 0.00837795 & 0.00282504 & 0.000825997 & 0.000225008 & $5.90462 e-05$ \\
\hline & Order & 1.568326 & 1.774063 & 1.876160 & 1.930060 \\
\hline \multirow{2}{*}{$\|E\|_{1}$} & 0.0279984 & 0.00950852 & 0.00280233 & 0.00077052 & 0.000205078 \\
\hline & Order & 1.558052 & 1.762594 & 1.862723 & 1.909660 \\
\hline \multirow{2}{*}{$\|e\|_{0}$} & 0.0341734 & 0.0123334 & 0.00376493 & 0.00105695 & 0.000283961 \\
\hline & Order & 1.470303 & 1.711876 & 1.832716 & 1.896142 \\
\hline \multirow{2}{*}{$\|e\|_{L^{\infty}}$} & 0.197857 & 0.107171 & 0.0453294 & 0.0170497 & 0.00610541 \\
\hline & Order & 0.884544 & 1.241396 & 1.410701 & 1.481586 \\
\hline \multirow{2}{*}{$\|\vartheta\|_{0}$} & 0.000215482 & $5.3851 e-05$ & $1.34612 e-05$ & $3.36511 e-06$ & $8.4091 e-07$ \\
\hline & Order & 2.000522 & 2.000166 & 2.000081 & 2.000630 \\
\hline \multirow{2}{*}{$\|\vartheta\|_{L^{\infty}}$} & 0.000197687 & $4.98479 e-05$ & $1.25012 e-05$ & $3.1314 e-06$ & $7.84502 e-07$ \\
\hline & Order & 1.987613 & 1.995466 & 1.997187 & 1.996959 \\
\hline \multirow{2}{*}{$\|\vartheta\|_{1}$} & 0.0167834 & 0.00839145 & 0.0041957 & 0.00209784 & 0.00104892 \\
\hline & Order & 1.000043 & 1.000009 & 1.000007 & 1.000000 \\
\hline
\end{tabular}

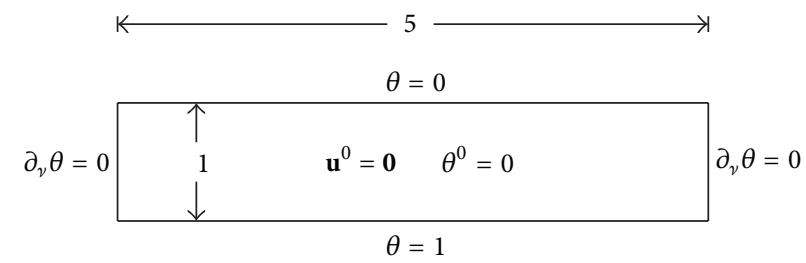

FIGURE 1: Example 20: initial and boundary values of Bénard convection problem.

Inserting the estimates from $A_{1}$ to $A_{7}$ back into (142) and employing the discrete inf-sup condition in Assumption 2, we obtain

$$
\begin{aligned}
& C\left\|e^{n+1}\right\|_{0} \\
& \leq \frac{1}{\tau}\left(\left\|\delta \mathbf{E}^{n+1}\right\|_{0}+\left\|\delta \mathbf{E}^{n}\right\|_{0}\right) \\
&+\frac{1}{\sqrt{h}}\left(\left\|\mathbf{E}^{n+1}\right\|_{0}+\left\|\mathbf{E}^{n}\right\|_{0}\right) \\
&+\left\|\delta q_{h}^{n+1}\right\|_{0}+\left\|\nabla \widehat{\mathbf{E}}_{h}^{n+1}\right\|_{0} \\
&+\left\|\delta \delta \mathbf{u}\left(t^{n+1}\right)\right\|_{1}+\left\|\widehat{\mathbf{E}}^{n+1}\right\|_{0} \\
&+\left\|R^{n+1}\right\|_{-1}+\left\|\delta \delta \theta\left(t^{n+1}\right)\right\|_{0} \\
&+\left\|29_{h}^{n}-\vartheta_{h}^{n-1}\right\|_{0} \\
&+\left\|2 \eta^{n}-\eta^{n-1}\right\|_{0} .
\end{aligned}
$$

If we now square it, multiply it by $\tau$, and sum over $n$ from 1 to $N$, then Lemmas 13,15 , and 17 derive (141).

\section{Numerical Experiments}

We finally document 3 computational performances of SGUM. The first is to check accuracy, and then the next 2 examples are physically relevant numerical simulations, the Bénard convection problem and the thermal driven cavity flow. We perform the last 2 examples under the same set within [2], but we conclude with different numerical simulation for the second test, the Bénard convection problem, from that of [2]. We impose Taylor-Hood $(\mathscr{P} 2-\mathscr{P} 1)$ in all 3 experiments.

Example 19 (mesh analysis). In this first experiment, we choose square domain $[0,1] \times[0,1]$ and impose forcing term the exact solution to become

$$
\begin{gathered}
u=\pi\left(t^{2}-t+1\right) x^{2}(1-x)^{2} \sin (2 \pi y), \\
v=-2\left(t^{2}-t+1\right) x(2 x-1)(x-1) \sin ^{2}(\pi y), \\
p=-\left(t^{2}-t-1\right) \cos (\pi x)\left(y^{2}+1\right), \\
\theta=\cos (t) \sin (\pi x) y(1-y) .
\end{gathered}
$$

Table 1 is error decay with $\mu=1$ and $\tau=h$. We conclude that the numerical accuracy of SGUM is optimal and consists with the result of Theorem 4 . 

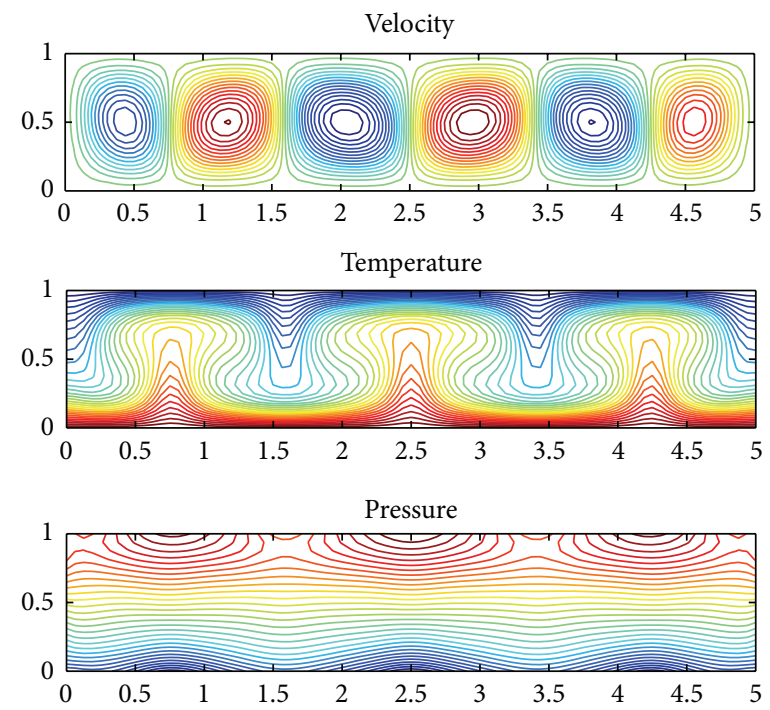

FIGURE 2: Example 20: streamlines of velocity and isolines of temperature and pressure, at time $t=1.0$. The nondimensional parameters are $\kappa=10^{4}, \lambda=1, \mu=1$, and the discretization parameters are $\tau=10^{-2}, h=2^{-4}$.
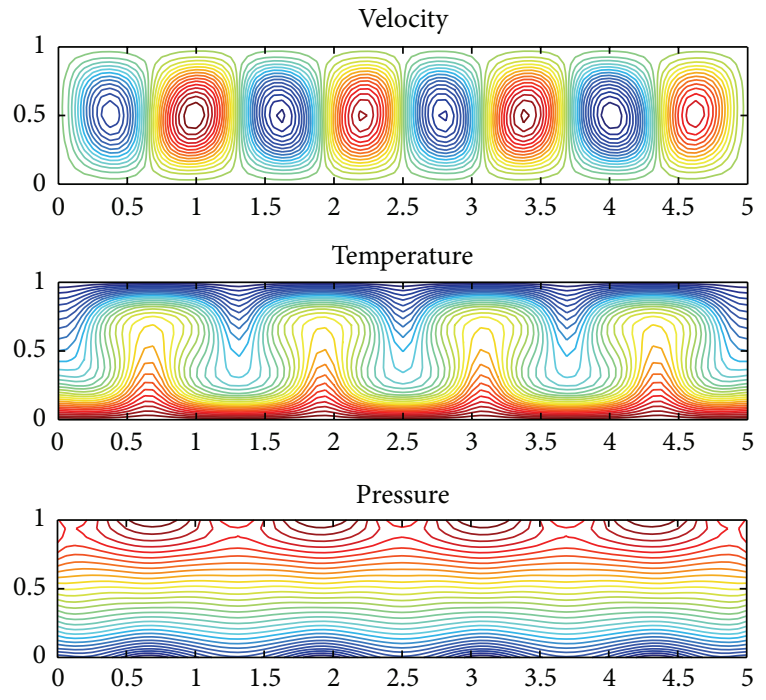

Figure 3: Example 20: streamlines of velocity and isolines of temperature and pressure, at time $t=1.0$. The nondimensional parameters are $\kappa=10^{4}, \lambda=1$, and $\mu=1$, and the discretization parameters are $\tau=10^{-3}, h=2^{-4}$.

Example 20 (Bénard convection). In order to explore the applicability of the SGUM, we consider the Bénard convection on the domain $\Omega=[0,5] \times[0,1]$ with forcing $\mathbf{f}=0$ and $b=0$. Figure 1 displays the initial and boundary conditions for velocity $\mathbf{u}$ and temperature $\theta$, as already studied in [2]. Figures $2-4$ are simulations at $t=1$ with the nondimensional parameters $\kappa=10^{4}, \lambda=1$, and $\mu=1$. Figure 2 is the result for the case $\tau=10^{-2}, h=2^{-4}$ which is the same condition in [2], and so it displays similar behavior within [2] including 6 circulations in the velocity stream line. However, Figures 3 and 4, the higher resolution simulations with $\tau=10^{-3}, h=$ $2^{-4}$, and $\tau=10^{-4}, h=2^{-6}$, display 8 circulations in
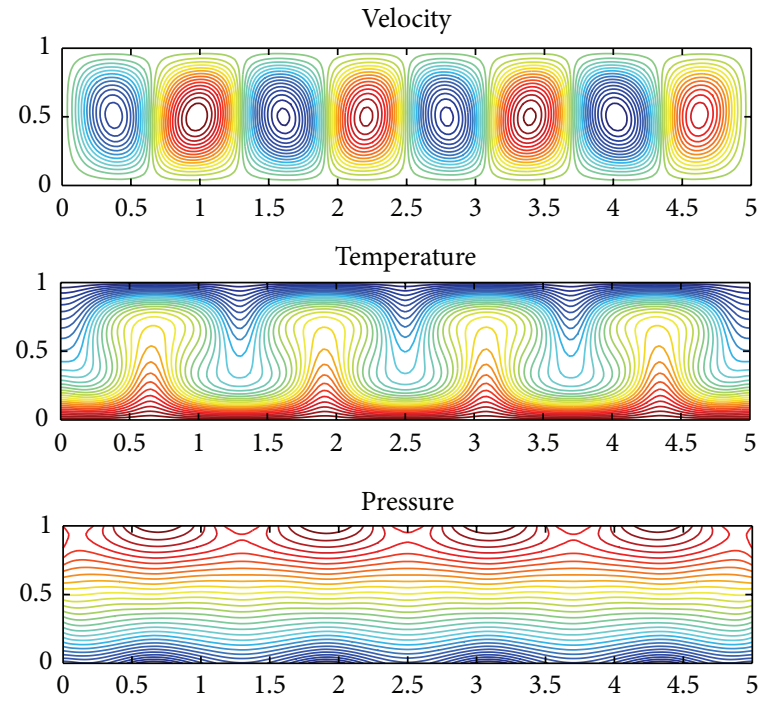

FIgURE 4: Example 20: streamlines of velocity and isolines of temperature and pressure, at time $t=1.0$. The nondimensional parameters are $\kappa=10^{4}, \lambda=1$, and $\mu=1$, and the discretization parameters are $\tau=10^{-4}, h=2^{-6}$.

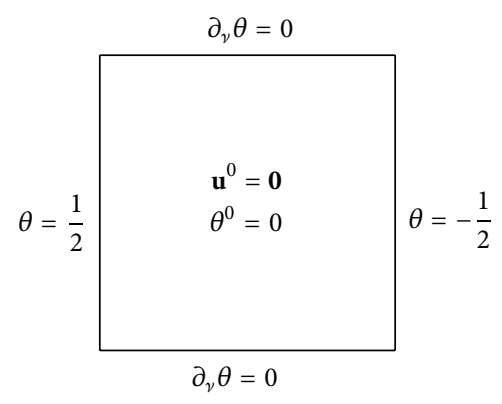

Figure 5: Example 21: initial and boundary values for thermal driven cavity flow. 

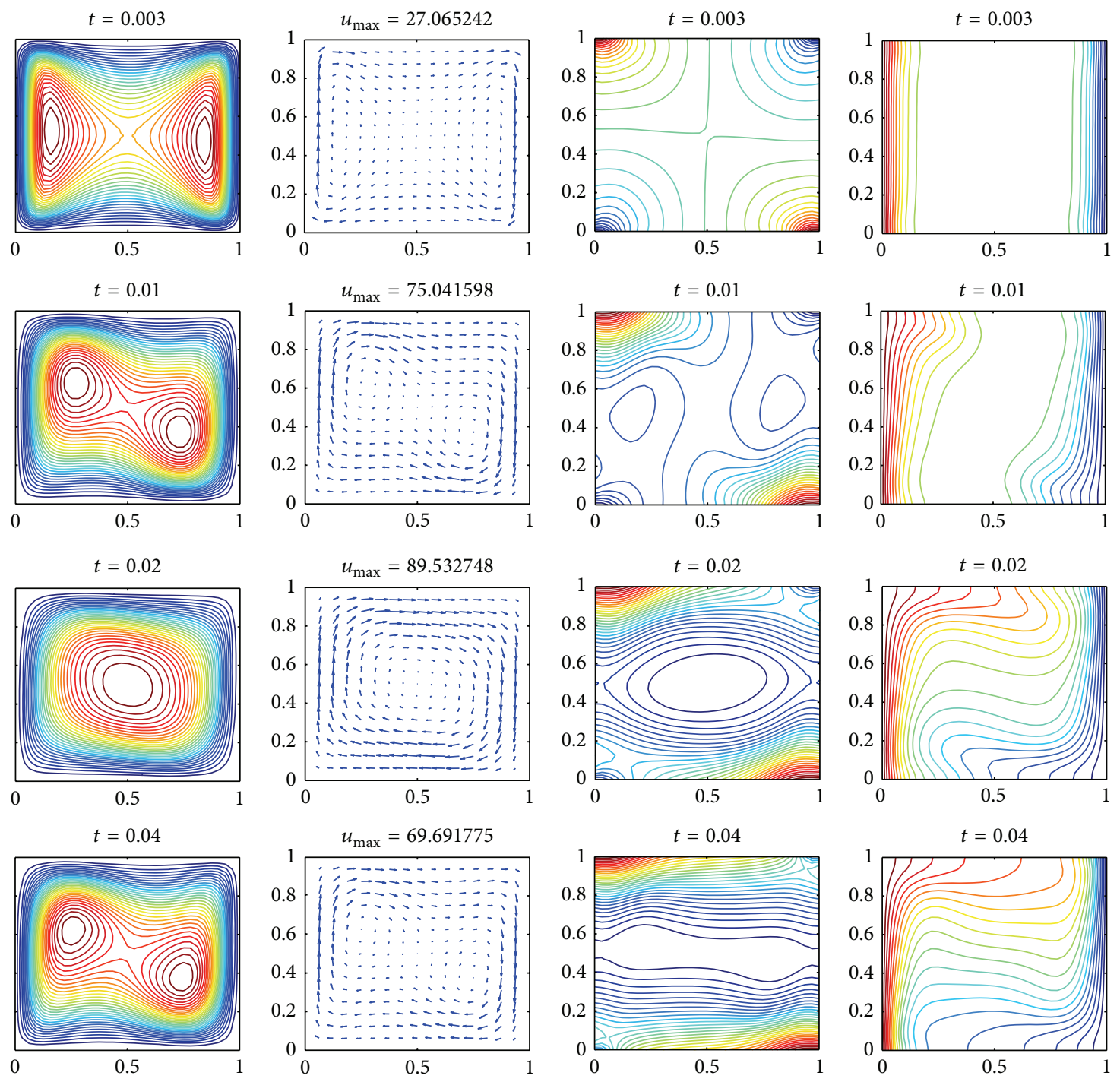

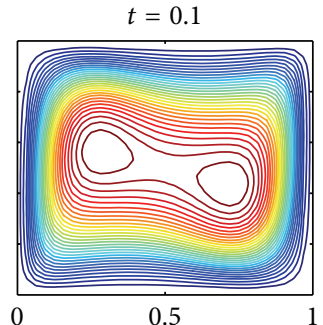

(a)

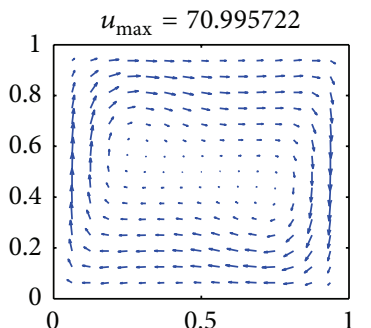

(b)

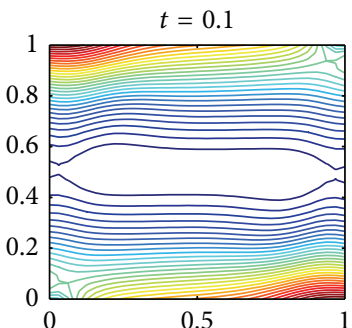

(c)

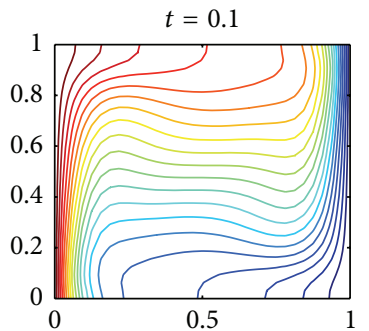

(d)

FIGURE 6: Example 21: time sequence $t=0.003,0.01,0.02,0.04$, and 0.1 for the driven cavity. The first two columns are the streamlines and vector fields for velocity, and the third and fourth ones are the contour lines for pressure and temperature, respectively. The nondimensional parameters are $\kappa=10^{5}, \lambda=1$, and $\mu=1$, and the discretization parameters are $\tau=10^{-4}, h=2^{-5}$. Note that $u_{\max }$ stands for $\|\mathbf{u}\|_{\infty}$.

the stream line. So we conclude that the high resolution result is correct simulation and thus Figure 2 and the result in [2] are not eventual simulation.

Example 21 (thermal driven cavity flow). We consider the thermal driven cavity flow in an enclosed square $\Omega=[0,1]^{2}$, as already studied in several papers $[2,6,7]$. The experiment is carried out with the same setting as in the work of
Gresho et al. [6], which is shown in Figure 5. Figure 6 displays the evolution from rest $(t=0)$ to steady state $(t=0.2)$.

\section{References}

[1] R. H. Nochetto and J.-H. Pyo, "The gauge-Uzawa finite element method. I. The Navier-Stokes equations," SIAM Journal on Numerical Analysis, vol. 43, no. 3, pp. 1043-1068, 2005. 
[2] R. H. Nochetto and J.-H. Pyo, "The gauge-Uzawa finite element method. II. The Boussinesq equations," Mathematical Models \& Methods in Applied Sciences, vol. 16, no. 10, pp. 1599-1626, 2006.

[3] J.-H. Pyo and J. Shen, "Gauge-Uzawa methods for incompressible flows with variable density," Journal of Computational Physics, vol. 221, no. 1, pp. 181-197, 2007.

[4] J.-H. Pyo and J. Shen, "Normal mode analysis of second-order projection methods for incompressible flows," Discrete and Continuous Dynamical Systems B, vol. 5, no. 3, pp. 817-840, 2005.

[5] J. H. Pyo, "Error estimates for the second order semi-discrete stabilized Gauge-Uzawa methodFor the Navier-Stokes equations," International Journal of Numerical Analysis \& Modeling, vol. 10, pp. 24-41, 2013.

[6] P. M. Gresho, R. L. Lee, S. T. Chan, and R. L. Sani, "Solution of the time-dependent incompressible Navier-Stokes and Boussinesq equations using the Galerkin finite element method," in Approximation Methods for Navier-Stokes Problems, vol. 771 of Lecture Notes in Mathematics, pp. 203-222, Springer, Berlin, Germany, 1980.

[7] K. Onishi, T. Kuroki, and N. Tosaka, "Further development of BEM in thermal fluid dynamics," in Boundary Element Methods in Nonlinear Fluid Dynamics, vol. 6 of Developments in Boundary Element Methods, pp. 319-345, 1990.

[8] S. C. Brenner and L. R. Scott, The Mathematical Theory of Finite Element Methods, Springer, 1994.

[9] F. Brezzi and M. Fortin, Mixed and Hybrid Finite Element Methods, Springer, 1991.

[10] V. Girault and P.-A. Raviart, Finite Element Methods for NavierStokes Equations, Springer, 1986.

[11] P. Constantin and C. Foias, Navier-Stokes Equations, The University of Chicago Press, Chicago, Ill, USA, 1988.

[12] J. G. Heywood and R. Rannacher, "Finite element approximation of the nonstationary Navier-Stokes problem. I. Regularity of solutions and second-order error estimates for spatial discretization," SIAM Journal on Numerical Analysis, vol. 19, no. 2, pp. 275-311, 1982.

[13] R. B. Kellogg and J. E. Osborn, "A regularity result for the Stokes problem in a convex polygon," Journal of Functional Analysis, vol. 21, no. 4, pp. 397-431, 1976.

[14] R. Temam, Navier-Stokes Equations, AMS Chelsea, 2001. 


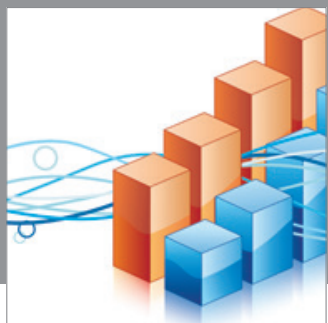

Advances in

Operations Research

mansans

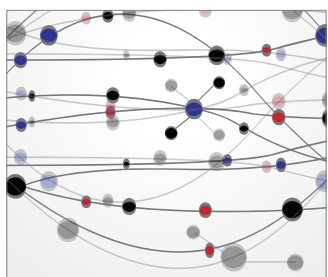

The Scientific World Journal
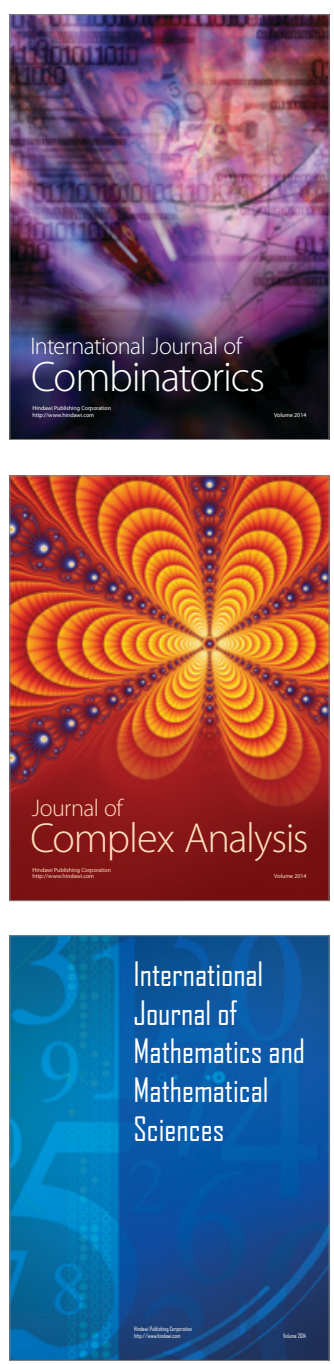
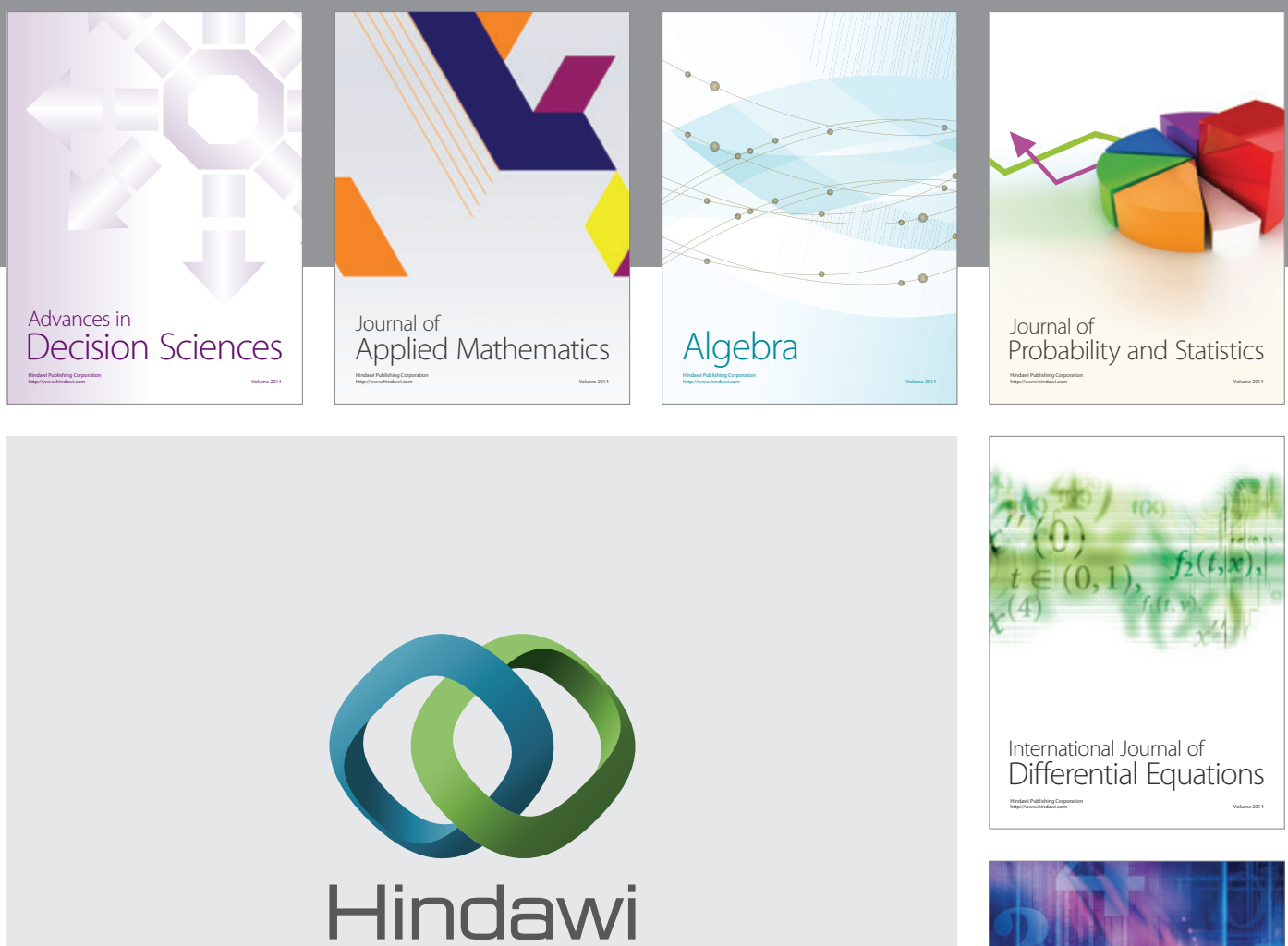

Submit your manuscripts at http://www.hindawi.com
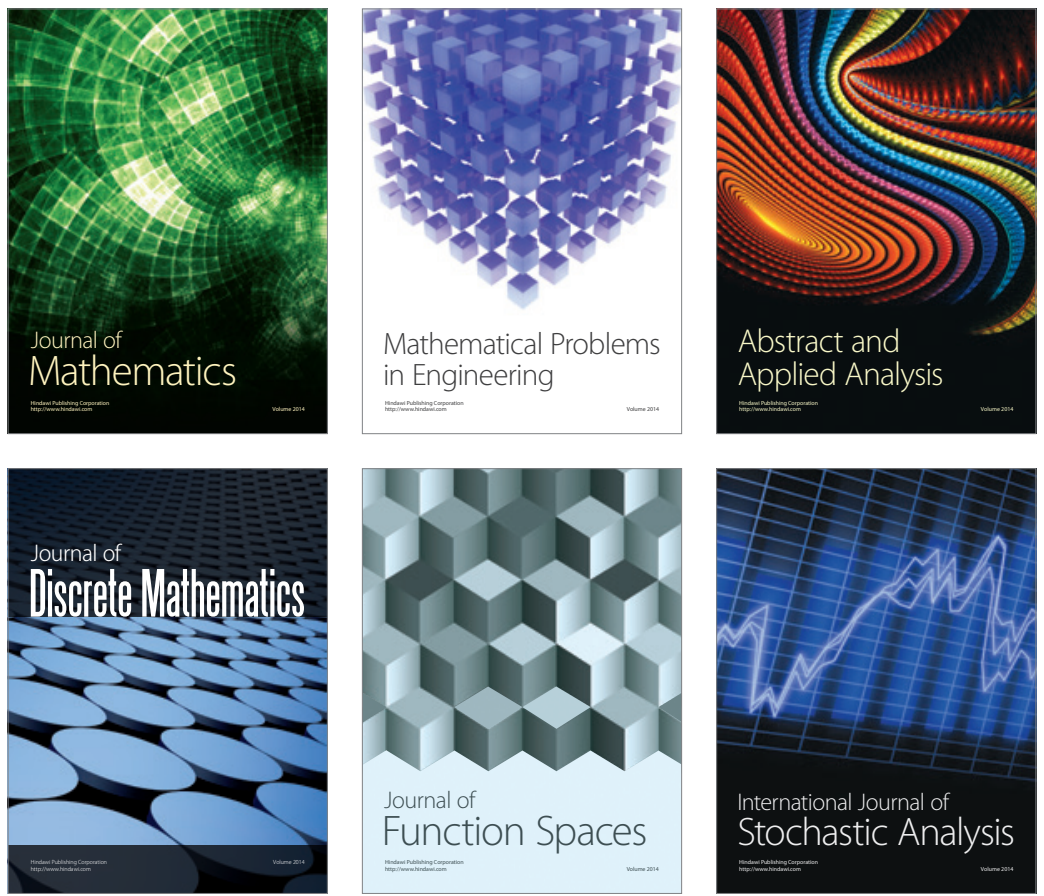

Journal of

Function Spaces

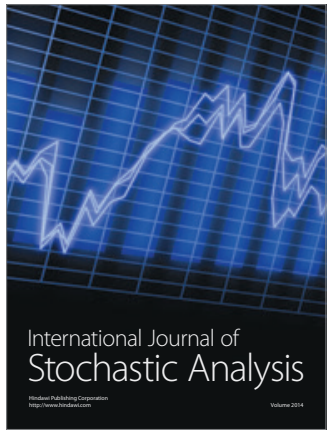

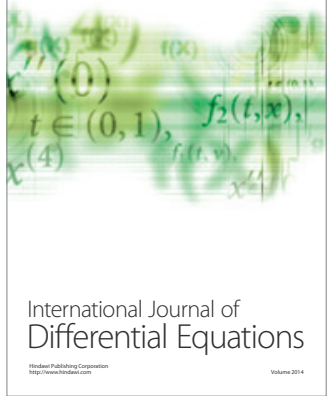
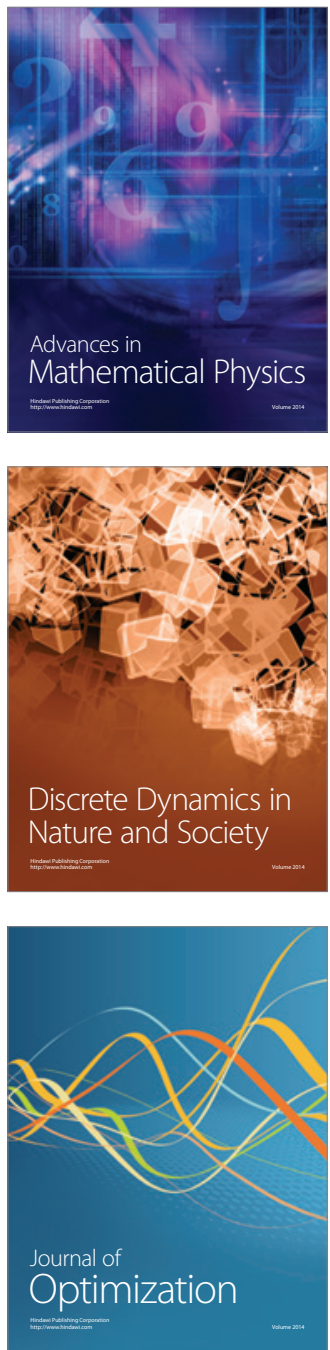